\title{
The impact of oceanic heat transport on the atmospheric circulation
}

\author{
M.-A. Knietzsch ${ }^{1}$, A. Schröder ${ }^{1}$, V. Lucarini ${ }^{1,2}$, and F. Lunkeit ${ }^{1}$ \\ ${ }^{1}$ Meteorologisches Institut, Universität Hamburg, Hamburg, Germany \\ ${ }^{2}$ Department of Mathematics and Statistics, University of Reading, Reading, UK \\ Correspondence to: F. Lunkeit (frank.lunkeit@uni-hamburg.de) \\ Received: 2 October 2014 - Published in Earth Syst. Dynam. Discuss.: 4 November 2014 \\ Revised: 28 August 2015 - Accepted: 3 September 2015 - Published: 21 September 2015
}

\begin{abstract}
A general circulation model of intermediate complexity with an idealized Earth-like aquaplanet setup is used to study the impact of changes in the oceanic heat transport on the global atmospheric circulation. Focus is on the atmospheric mean meridional circulation and global thermodynamic properties.

The atmosphere counterbalances to a large extent the imposed changes in the oceanic heat transport, but, nonetheless, significant modifications to the atmospheric general circulation are found. Increasing the strength of the oceanic heat transport up to $2.5 \mathrm{PW}$ leads to an increase in the global mean near-surface temperature and to a decrease in its equator-to-pole gradient. For stronger transports, the gradient is reduced further, but the global mean remains approximately constant. This is linked to a cooling and a reversal of the temperature gradient in the tropics.

Additionally, a stronger oceanic heat transport leads to a decline in the intensity and a poleward shift of the maxima of both the Hadley and Ferrel cells. Changes in zonal mean diabatic heating and friction impact the properties of the Hadley cell, while the behavior of the Ferrel cell is mostly controlled by friction.

The efficiency of the climate machine, the intensity of the Lorenz energy cycle and the material entropy production of the system decline with increased oceanic heat transport. This suggests that the climate system becomes less efficient and turns into a state of reduced entropy production as the enhanced oceanic transport performs a stronger large-scale mixing between geophysical fluids with different temperatures, thus reducing the available energy in the climate system and bringing it closer to a state of thermal equilibrium.
\end{abstract}

\section{Introduction}

The climate is a forced and dissipative nonequilibrium system, which - neglecting secular trends - can be considered to be in steady state, i.e., its statistical properties do not depend on time. Astronomical factors and differences in local albedo cause a difference in net incoming shortwave radiation between low and high latitudes leading to differential heating and a surplus of energy in the tropics. Over a global and long-term average, all supplied energy is emitted to space, so that the incoming shortwave radiation is balanced by the outgoing longwave radiation (Peixoto and Oort, 1992; Lucarini and Ragone, 2011). The ocean and atmosphere transport the excess of energy from the tropics to high latitudes so that the horizontal divergence of the large-scale transport performed by the geophysical fluids on the average balances out the radiative imbalances at the top of the atmosphere.

The oceanic and atmospheric transports result from the conversion of available potential energy - due to the inhomogeneous absorption of solar radiation, with a positive correlation between heating and temperature patterns - into kinetic energy, through instabilities arising, typically, from the presence of temperature gradients (Lorenz, 1955). Such instabilities tend to reduce the same temperature gradients they feed upon by mixing oceanic and atmospheric masses. The kinetic energy is then dissipated inside the system. The production of available potential energy, its conversion to kinetic energy, and the dissipation of kinetic energy have the same average rate, which corresponds to the intensity of the Lorenz $(1955,1967)$ energy cycle. 
Recently, using tools of macroscopic nonequilibrium thermodynamics, a connection has been drawn between a measure of the efficiency of the climate system, the spatiotemporal variability in its heating and temperature fields, the intensity of the Lorenz energy cycle and the material entropy production (Johnson, 2000; Lucarini, 2009; Lucarini et al., 2011). As mentioned above, the climate can be considered as a (forced and dissipative) nonequilibrium thermodynamic system where the entropy budget is achieved in such a way that the sum of the incoming entropy flux due to the solar high-frequency photons, and the entropy generated by irreversible processes in the atmosphere and ocean, is balanced out by the radiation to space of low-frequency photons. Most of the entropy production results from optical processes, while a smaller portion - referred to as material entropy production - is related to the irreversible processes related to diffusion and dissipation taking place in geophysical fluids (Kleidon and Lorenz, 2005). So the Earth is, in contrast to a system that is isolated and therefore maintained in a state of equilibrium, a thermodynamic system that exchanges energy and entropy with space (Ambaum, 2010).

Stone (1978) argued that the magnitude of the total meridional heat transport, i.e., the sum of the oceanic and the atmospheric contributions, is almost insensitive to the structure and the specific dynamical properties of the atmosphereocean system, so that changes in the oceanic heat transport (OHT) will be balanced out by the atmospheric flow and vice versa. In particular, he suggested that the peak of the heat transport is constrained within a narrow range of latitudes regardless of the radiative forcing. Stone concluded that features of the meridional heat transport can be related to the solar constant, the radius of the Earth, the tilt of the Earth's axis and the hemispheric mean albedo. He argued that the insensitivity to the structure and to the dynamics of the system is due to the correlation of thermal emissions to space, the albedo and the efficiency of the transport mechanisms of the atmosphere and the ocean.

Enderton and Marshall (2008) discussed the limits of the Stone (1978) hypothesis by employing a series of coupled atmosphere-ocean-sea-ice model experiments in which the oceanic circulation on an aquaplanet is constrained by different meridional barriers. The presence or absence of the barriers results in significantly different climates, in particular in climates with and without polar ice caps. Enderton and Marshall concluded that Stone's result is a good guide for ice-free climates. However, they also noted that the effect of the related meridional gradients in albedo on the absorption of solar radiation needs to be taken into account if polar ice caps are present.

The atmospheric compensation implies a significant impact of changes in OHT on the atmospheric circulation as a whole. These changes in the atmospheric circulation concern the zonally symmetric flow, the zonally asymmetric (eddy) flow and the interplay between both. Thus, changes in OHT have been commonly used to account for paleocli- matic changes (e.g., Rind et al., 1991; Sloan et al., 1995; Romanova et al., 2006). Moreover, OHT is an important factor for potential anthropogenic climate change since significant modifications to it can be expected. Unfortunately, there are large uncertainties in the changes in the oceanic circulation simulated in climate change scenarios (IPCC, 2013). These result from, amongst others, uncertainties in freshwater forcing due to potential melting of inland ice sheets. To assess the role of the ocean for historical and potential future climates the impact of the OHT on the atmospheric circulation and the underlying mechanisms need to be investigated systematically.

A way of studying the impact of changes in OHT on the atmospheric circulation is to utilize an atmospheric general circulation model coupled to a mixed-layer ocean. In such a model the OHT can be prescribed. Using a present-day setup including continents, Winton (2003), Herweijer et al. (2005), and Barreiro et al. (2011) found that increasing OHT results in a warmer climate with less sea ice. A reduction in lowlevel clouds and an increase in greenhouse trapping due to a moistening of the atmosphere appeared to be relevant mechanisms. In addition, a weakening of the Hadley cell with increased OHT was found by Herweijer et al. (2005) and Barreiro et al. (2011).

Utilizing an idealized aquaplanet setup, Rose and Ferreira (2013) systematically assessed the impact of the OHT on the atmospheric global mean near-surface temperature and its equator-to-pole gradient. For warm and ice-free climates, they confirm a near-perfect atmospheric compensation of the imposed changes in OHT. Like in the above studies including continents, Rose and Ferreira (2013) found an increase in global mean temperature for increasing OHT, accompanied by a decrease in the equator-to-pole temperature gradient. Tropical SSTs (sea surface temperatures) were shown to be less affected than those at higher latitudes. The detailed meridional structure of the oceanic heat transport turned out to be less important. Changes in deep moist convection in the midlatitudes, together with an enhanced water vapor greenhouse effect, appear to be the major drivers. Koll and Abbot (2013) confirmed the low sensitivity of tropical SSTs to OHT changes. In their aquaplanet experiments, larger OHT leads to a weakening of the Hadley cell, which reduces cloud cover and surface winds and thus counteracts surface cooling resulting from increasing OHT.

In the present study we extend and supplement the above studies. Based on the experimental setup of Rose and Ferreira (2013), we focus on the impact of OHT changes on the atmospheric dynamics and thermodynamics. Our overall goal is to understand how the atmospheric energy transport and transformations are affected by modulations in the oceanic transport. We analyze the changes in the atmospheric heat transport and the mean meridional circulation by employing, amongst others, the Kuo-Eliassen equation (Kuo, 1956; Eliassen, 1951) in order to understand the various drivers of the mean meridional circulation. 
Furthermore, the integrated effect on the global atmospheric energetics is assessed by changes in the properties of the effective warm and cold reservoirs constructed according to the theory proposed in Johnson (2000) and Lucarini (2009). This allows for defining a measure of the efficiency of the climate system viewed as an (equivalent Carnot) engine. Attention is directed to measuring the irreversibility of the atmosphere and the material entropy production. This point of view is useful for providing a general physical framework to relevant climatic processes, trying to advance the understanding of the climate as a nonequilibrium, forced and dissipative macroscopic system. Links between the climate engine view and the classical Lorenz energy cycle (Lorenz, 1955) provide a consistent picture of the observed changes and document the relevance of the climate engine approach.

The paper is organized as follows. In Sect. 2 we describe the model and the experimental design. Section 3 introduces our diagnostics. The results of the analyses are presented in Sect. 4. A summary and discussion concludes the paper (Sect. 5). Appendices A-C give comprehensive descriptions of the main diagnostics.

\section{Model and experimental setup}

The Planet Simulator (PlaSim; Fraedrich et al., 2005) is an open-source general circulation model (GCM) of intermediate complexity developed at the University of Hamburg. For the atmosphere, the dynamical core is the Portable University Model of the Atmosphere (PUMA) based on the primitiveequation multilevel spectral model of Hoskins and Simmons (1975) and James and Gray (1986). Radiation is parameterized by differentiating between shortwave and longwave radiation and between a clear and a cloudy atmosphere. The respective schemes follow the works of Lacis and Hansen (1974) for the shortwave part and Sasamori (1968) for the longwave part. The radiative properties of clouds are based on Stephens (1978) and Stephens et al. (1984). Cloud fraction is computed according to Slingo and Slingo (1991). The representation of boundary-layer fluxes and of vertical and horizontal diffusion follows Louis (1979), Louis et al. (1982), Roeckner et al. (1992), and Laursen and Eliasen (1989). The cumulus convection scheme is based on Kuo $(1965,1974)$. The ocean is represented by a thermodynamic mixed-layer (slab ocean) model including a one-layer thermodynamic sea-ice component.

Following Rose and Ferreira (2013) we used an Earthlike aquaplanet setup with zonally symmetric forcing utilizing reference present-day conditions for the solar constant $\left(1365 \mathrm{~W} \mathrm{~m}^{-2}\right)$ and the $\mathrm{CO}_{2}$ concentration $(360 \mathrm{ppm})$. The solar insolation comprises an annual cycle (with obliquity $=23.4^{\circ}$ ), but eccentricity is set to 0 . Thus, on annual average the forcing is hemispherically symmetric as well. The mixed-layer depth is set to $60 \mathrm{~m}$.

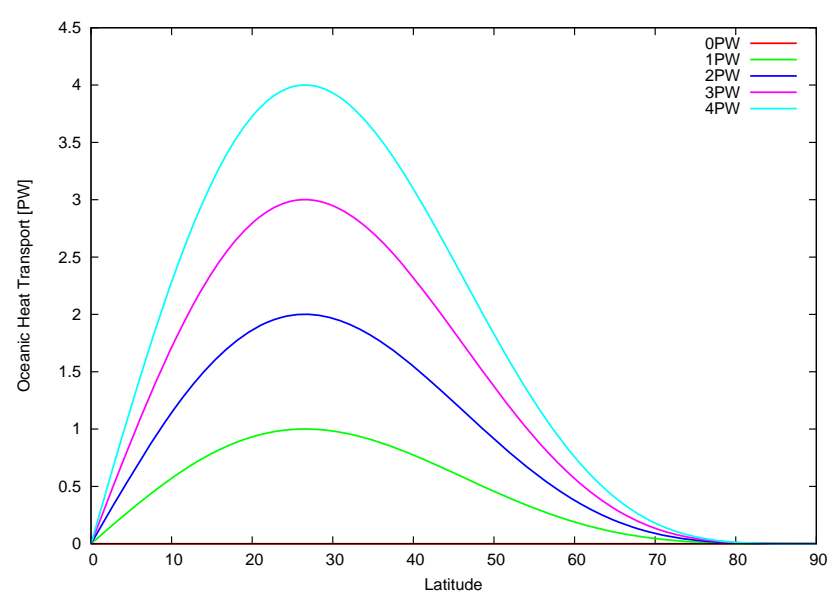

Figure 1. Oceanic heat transport (in PW) for $\mathrm{OHT}_{\max }=0,1,2,3$, and $4 \mathrm{PW}$.

A temporally constant flux into the ocean ( $q$-flux) is used to prescribe the oceanic heat transport (OHT) according to the analytic expression given by Rose and Ferreira (2013):

$\mathrm{OHT}=\mathrm{OHT}_{0} \cdot \sin (\phi) \cos (\phi)^{2 N}$,

where $\phi$ denotes the latitude. $N$ is a positive integer which determines the latitude of the maximum of the transport and the shape of its meridional profile, and $\mathrm{OHT}_{0}$ is a constant defining its magnitude. Rose and Ferreira made sensitivity experiments by varying $N$ (ranging from 1 to 8 ) and by varying the peak transport (ranging from 0 to $4 \mathrm{PW}$ ), which is controlled by $\mathrm{OHT}_{0}$.

For our study we follow Rose and Ferreira but fix the location of the peak by setting $N=2$ (which corresponds to maximum transport at $27^{\circ}$ ). We perform nine sensitivity simulations with respect to the magnitude of the transport by changing $\mathrm{OHT}_{0}$ to obtain peak transports $\mathrm{OHT}_{\max }$ from 0 to $4 \mathrm{PW}$ (with $0.5 \mathrm{PW}$ increment). $\mathrm{OHT}_{\max }=0 \mathrm{PW}$ (i.e., no OHT) serves as the control simulation. The OHT for $\mathrm{OHT}_{\max }=0$, 1, 2, 3 and 4 PW is displayed in Fig. 1.

All simulations are run for at least 100 years (360 days per year). The last 30 years are subject to the analyses. A horizontal resolution of $T 31$ ( $96 \times 48$ grid points) with five $\sigma$ levels in the vertical is used. The time step is $\Delta t=23 \mathrm{~min}$.

\section{Diagnostics}

The dominant feature of the large-scale ocean and the atmosphere dynamics is the transport of energy from regions featuring a net positive energy budget at the top of the atmosphere low latitudes) to regions where such a budget is negative (high latitudes). This reduces the temperature gradient between the equator and the poles (e.g., Peixoto and Oort, 1992; Lucarini and Ragone, 2011). In present conditions, the partitioning of heat transport between atmosphere and ocean reflects two limits: the dominance of the atmospheric 
mass transport in mid- to high latitudes and the dominance of the oceanic energy transport in the tropics. The atmospheric transport can be further subdivided in the sensibleheat, latent-heat and potential-energy components. We will investigate the response of changes in the imposed OHT for each of these components and will further focus the analysis by considering both the zonally symmetric contributions, due to the mean meridional circulation (MMC) and the zonally asymmetric contributions, due to the atmospheric eddies.

In the classical view (the Eulerian mean circulation), the mean meridional circulation consists of three cells: the tropical Hadley cell, the Ferrel cell in midlatitudes and a weak polar cell. While the Hadley and the polar cell are thermally direct circulations, i.e., relatively warm air is rising and cold air is sinking, the Ferrel cell is referred to as a thermally indirect cell with warm air sinking and cold air rising. Though the mean meridional circulation can be viewed as a two dimensional circulation in the meridional-height plane, both zonally symmetric and zonally asymmetric components contribute to its existence.

The transformed Eulerian mean (TEM) formalism (Andrews and McIntyre, 1976) accounts for the role of the eddies in the mean meridional transport. In particular, it provides a closer link to the total atmospheric meridional heat transport. The TEM residual circulation approximates the (dry) isentropic mean circulation resulting in a single cell from the equator to the pole.

Based on work by Held (2001), Czaja and Marshall (2006) showed that the atmospheric heat transport can be represented by the product of a moist TEM residual circulation and the vertical contrast in moist static energy (or equivalent potential temperature $\theta_{\mathrm{e}}$ ). The moist residual circulation is given by replacing all terms containing dry static energy (or potential temperature) by their moist analogs.

Utilizing the Kuo-Eliassen equations allows for identifying individual drivers of the Eulerian mean meridional circulation (Appendix A). A similar partitioning is done for TEM residual stream function which provides a direct link to the atmospheric heat transport. However, Pauluis et al. (2011) and Laliberté et al. (2012) pointed out that there is no simple way to represent a well-defined moist isentropic circulation in the latitude-pressure plane. Therefore, in order to assess the effect of moisture, we additionally investigate the mean circulation on dry and moist isentropes.

This summarizes the diagnostics tools aimed at capturing a phenomenological description of the atmospheric circulation.

A second set of diagnostic tools is based on taking a thermodynamical point of view on the atmospheric circulation. One finds that on average a net positive work resulting from the positive correlation between temperature and heating fields upholds the kinetic energy of the global circulation against the frictional dissipation (Peixoto and Oort, 1992).

The atmospheric energy cycle (Lorenz, 1955, 1967) is one of the most important concepts used to understand the global atmospheric circulation as it provides a comprehensive look at the integrated effects of physical mechanisms involved, the generation of available potential energy by external forcing, the dissipation of kinetic energy and the energy conversions by baroclinic and barotropic processes. If the climate system is in a statistical steady state, the rate of generation of available potential energy $\dot{G}$, the rate of conversion of potential into kinetic energy $\dot{W}$, and the dissipation rate of kinetic energy $\dot{D}$ are equal when averaged over a long period of time (e.g., several years). Thus, $\overline{\dot{G}}=\overline{\dot{W}}=\overline{\dot{D}}>0$, where the bar indicates the operation of time averaging. This allows for characterizing the strength of the energy cycle in several ways.

Following the work by Johnson (2000) and Lucarini (2009), we consider the global energy cycle as resulting from the work of an equivalent Carnot engine operating between the two (dynamically determined) reservoirs at temperature $\Theta^{+}$and $\Theta^{-}$. According to this concept, an efficiency of the climate system $(\eta)$ can be defined by

$\eta=\frac{\Theta^{+}-\Theta^{-}}{\Theta^{+}}$.

Furthermore, following the same theoretical point of view, we analyze the entropy production which leads to a measure of the irreversibility of the climatic processes. An outline of the theory and the according diagnostics is given in Appendix B.

The diagnostics of the Lorenz formulation of the energy cycle reveal information about the reservoirs partitioned into zonal mean and eddy components and about the conversions due to different physical processes (Appendix C). We gain evidence about the relative importance of the individual components contributing to the energy cycle and the related thermodynamic properties. Furthermore, the classical Lorenz energy cycle helps to provide a link between the phenomenological view (given by the circulation and transports) and the thermodynamic view (the equivalent Carnot engine), thus demonstrating the relevance of the latter.

\section{Results}

\subsection{Mean climate}

We start with the discussion of the effect of OHT changes on the mean climate in terms of atmospheric near-surface $(2 \mathrm{~m})$ temperature, sea-ice and meridional heat transport. First, we note that, similarly to Rose and Ferreira (2013), our model exhibits multiple equilibria, a warm state and a snow-ball Earth depending on the initial conditions as thoroughly discussed in Boschi et al. (2013) and Lucarini et al. (2013). In the present study we investigate the warm states only. However, in contrast to Rose and Ferreira (2013), sea ice at high latitudes is present in all of the warm-state simulations.

$\mathrm{Up}$ to about $\mathrm{OHT}_{\max }=2.5 \mathrm{PW}$ increasing OHT leads to an increase in the global mean $\left(T_{\mathrm{M}}\right)$ and a decrease in the equator-to-pole gradient $(\Delta T)$ of the annual and zonal mean 

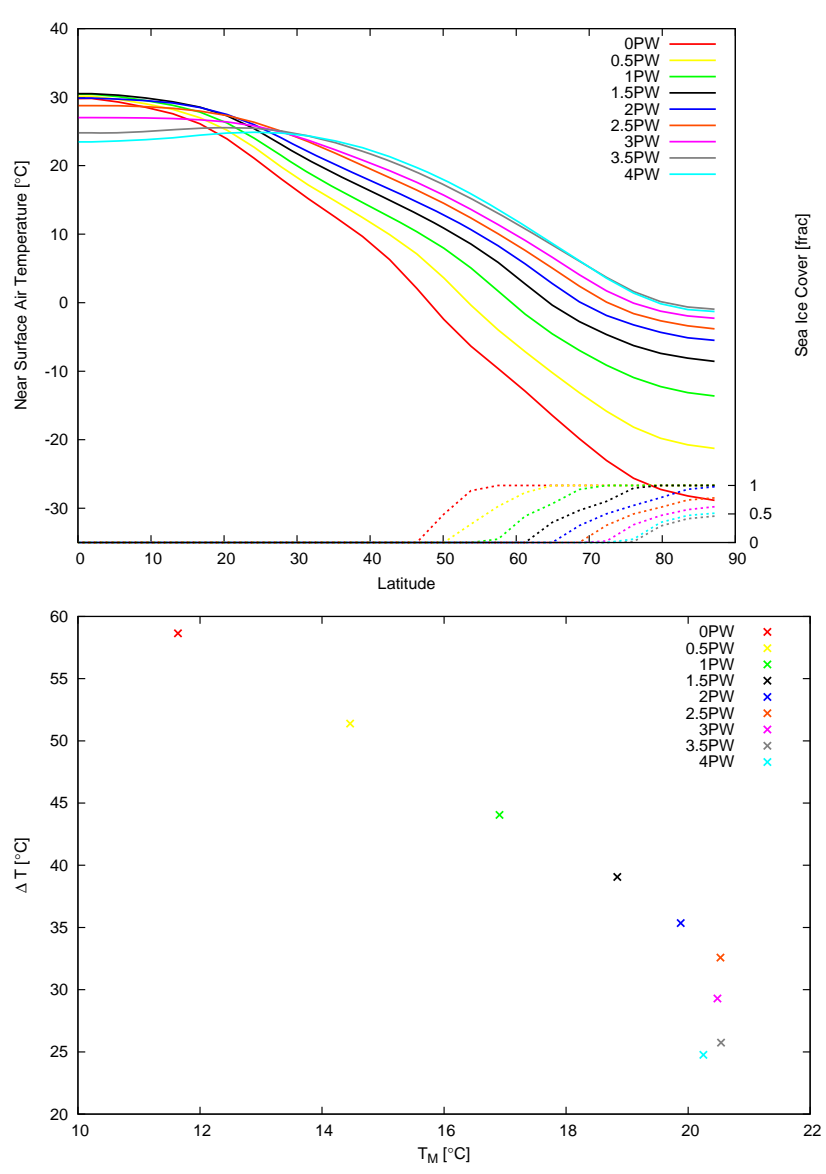

Figure 2. Climatological annual averages for all simulations. Upper panel: zonal mean near-surface temperature (solid lines) and sea-ice cover (dotted lines). Lower panel: global mean near-surface temperatures $\left(T_{\mathrm{M}}\right.$, in $\left.{ }^{\circ} \mathrm{C}\right)$ vs. equator-to-pole gradient $\left(\Delta T\right.$, in $\left.{ }^{\circ} \mathrm{C}\right)$.

near-surface air temperature (Fig. 2). For this regime an approximately linear relationship between $T_{\mathrm{M}}$ and $\Delta T$ can be found. For $\mathrm{OHT}_{\max }>2.5 \mathrm{PW}, T_{\mathrm{M}}$ is almost insensitive to an OHT change, while $\Delta T$ is further reduced with increasing intensity of transport. Here, the equator-to-pole gradient is defined by the difference between the values at the lowest and highest latitude of the model's grid which are located at about 0.9 and $85.8^{\circ}$, respectively.

When inspecting the meridional profiles of the annual and zonal mean near-surface temperatures, we observe that high latitudes are more sensitive to the OHT changes than low latitudes. With increasing OHT, polar temperatures continuously increase except that for $\mathrm{OHT}_{\max }=4 \mathrm{PW}$ slightly colder polar temperatures than for $\mathrm{OHT}_{\max }=3.5 \mathrm{PW}$ are found. It appears that this is a consequence of the reduced atmospheric heat transport, slightly overcompensating for the increased but still small oceanic heat transport at these latitudes (see later discussions). In the tropics, an increase in the temperatures is only present until $\mathrm{OHT}_{\max }=1.5 \mathrm{PW}$. For larger OHT, the equatorial temperatures decrease. In addi-

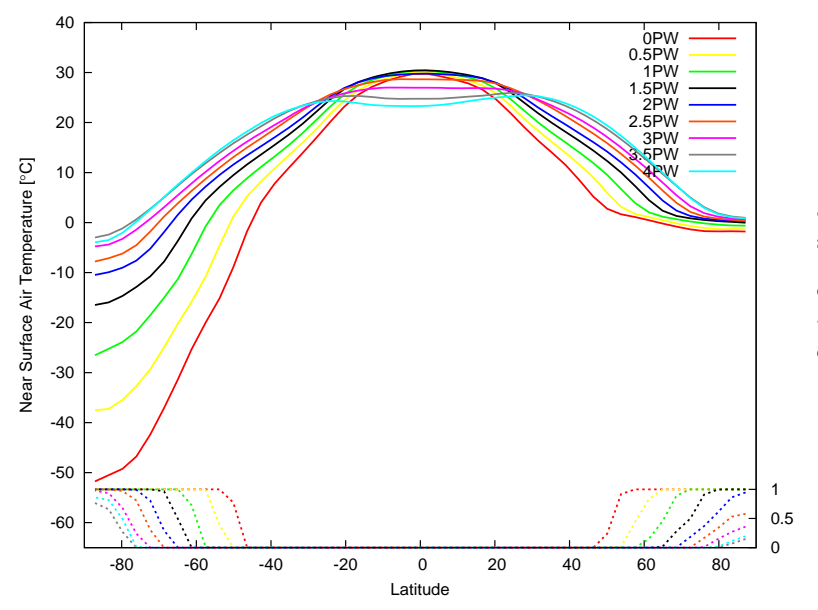

Figure 3. Climatological Northern Hemisphere summer (JuneAugust) averages for all simulations: zonal mean near-surface temperatures (in ${ }^{\circ} \mathrm{C}$; solid lines) and sea-ice cover (dotted lines).

tion, increasing OHT leads to a flattening of the temperature profile in the tropics until, for $\mathrm{OHT}_{\max }=3.5$ and $4 \mathrm{PW}$, the temperature gradient in the tropics gets reversed and the maximum of the temperature shifts away from the equator to approx. $\pm 24^{\circ}$.

Sea ice gradually decreases with increasing OHT. However, even for $\mathrm{OHT}_{\max }=4 \mathrm{PW}$ some sea ice remains in polar latitudes. However, for $\mathrm{OHT}_{\max }>2 \mathrm{PW}$ the average sea-ice cover is smaller than 1, indicating that no latitude is completely covered by sea ice during the whole year.

Qualitatively, all findings are also true for winter and summer as can be seen in Fig. 3, except that in summer the sensitivity to OHT changes is small for the sea-ice-covered high latitudes. In addition, we note that the seasonality and its sensitivity to OHT changes are small for latitudes without sea ice due to the high thermal inertia of the mixed layer. In the following we restrict the analysis to the annual mean.

Despite the difference in sea-ice extent (i.e., planetary albedo), the atmospheric heat transport compensates for the changes in OHT to a large extent, as can be inferred from the small differences in total meridional heat transport diagnosed from the energy budget at the top of the atmosphere (Fig. 4).

\subsection{Thermodynamics}

Now we shift our attention to the global thermodynamical properties of the system and investigate how the energetics and the entropy budget are impacted by changes in the imposed meridional oceanic heat transport.

\subsubsection{Efficiency}

As thoroughly discussed in Appendix B, the generation of available potential energy that powers the global atmospheric circulation results from the presence of a positive spatial and 


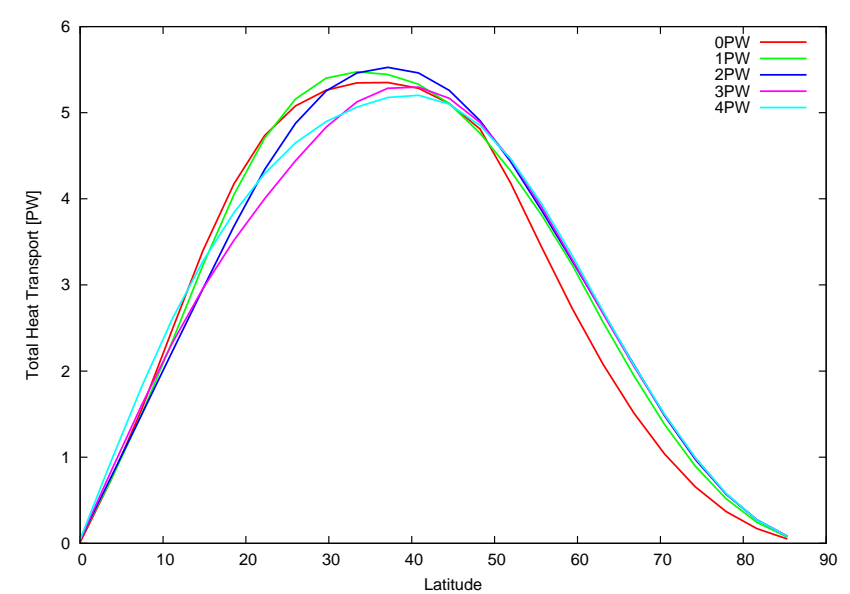

Figure 4. Total heat transport (in PW) diagnosed from energy budget at the top of the atmosphere for $\mathrm{OHT}_{\max }=0,1,2,3$, and $4 \mathrm{PW}$.

temporal correlation between the heating and the temperature fields. As a result, one can introduce two temperatures, $\Theta^{+}$and $\Theta^{-}$, which characterize the warm and cold reservoirs of the system, in such a way that the total intensity of the Lorenz energy cycle $\overline{\dot{W}}$ can be expressed as the product of the thermodynamic efficiency $\eta=\left(\Theta^{+}-\Theta^{-}\right) / \Theta^{+}$of the climate engine times the net heating of the warm reservoir. We first look into the sensitivity of $\Theta^{+}, \Theta^{-}$, and $\eta$ with respect to changes in the OHT. The relation to the Lorenz energy cycle will be discussed later in Sect. 4.4.

Qualitatively, $\Theta^{+}$and $\Theta^{-}$behave similarly when $\mathrm{OHT}_{\max }$ is changed (Fig. 5). We can classify three temperature regimes: (i) $\mathrm{OHT}_{\max }<2.0 \mathrm{PW}$ atmospheric warming, (ii) $2.0 \mathrm{PW} \leq \mathrm{OHT}_{\max } \leq 3.5 \mathrm{PW}$ atmospheric cooling, and (iii) $\mathrm{OHT}_{\max }>3.5 \mathrm{PW}$ weak sensitivity. We observe a higher sensitivity of $\Theta^{-}$than $\Theta^{+}$for (i) which is generally due to the amplified polar warming. The difference between $\Theta^{+}$and $\Theta^{-}$, denoted as $\Delta \Theta$, decreases with increasing $\mathrm{OHT}_{\max }$, thus implying a decrease in the atmospheric efficiency of the climate engine (Eq. 2). Interestingly, the difference between $T_{\mathrm{M}}$ and the average of $\Theta^{-}$and $\Theta^{+}$increases with $\mathrm{OHT}_{\max }$, especially for $\mathrm{OHT}_{\max } \leq 3.0 \mathrm{PW}$, indicating a reduction in the stability of the atmosphere. This is understood by considering that larger oceanic transports lead to stronger warming at low levels in the mid- and high latitudes, which must be balanced out by a weaker heat transport aloft.

The diabatic heating processes constitute the sources and sinks of internal energy for the atmosphere and play a decisive role in the generation and destruction of available potential energy (Peixoto and Oort, 1992). Those processes are displayed as the time- and zonally averaged diabatic heating rates $\mathrm{d} T_{\mathrm{a}} / \mathrm{d} t$ (Fig. 6). The heating rate is calculated as the sum over all diabatic heating effects including heating or cooling by the response of radiative heat fluxes, sensible and latent heat fluxes and vertical diffusion. While $\Theta^{+}$and $\Theta^{-}$are defined using the time- and space-dependent heating fields, in-

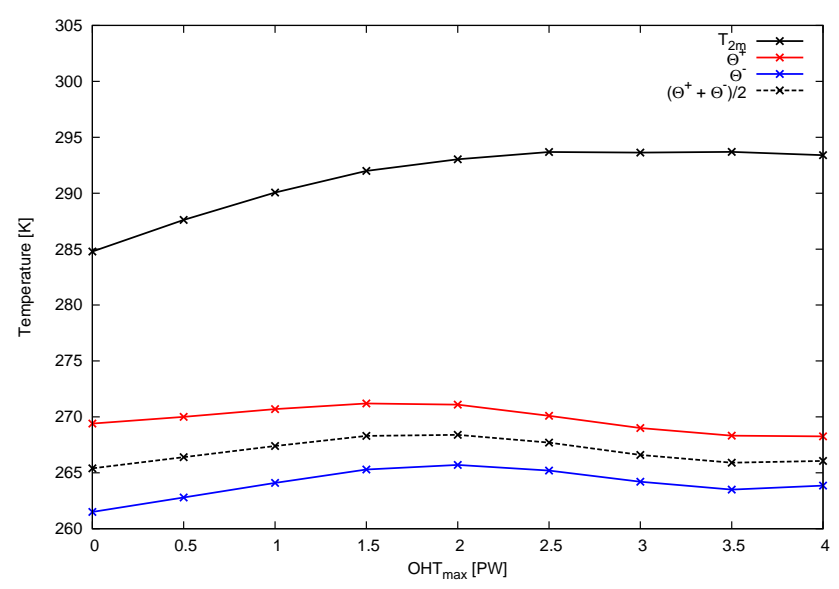

Figure 5. Time average of the global mean near-surface temperature $T_{\mathrm{M}}$ and of the temperature of the warm $\left(\Theta^{+}\right)$and the cold $\left(\Theta^{-}\right)$ pool.

specting the time and zonal averages of the heating patterns is useful for understanding how available potential energy is generated (Lucarini et al., 2010a).

Simulations with $0.5 \mathrm{PW} \leq \mathrm{OHT}_{\max } \leq 1.5 \mathrm{PW}$ show diabatic warming in the deep tropics, in the mid-troposphere and in the subtropical low troposphere, whereas diabatic cooling occurs in the mid- and high troposphere of the subtropics and in polar as well as subpolar regions. Positive heating in the tropical and subtropical regions is dominated by the contribution of latent heat fluxes, in particular, heating through convective precipitation (not shown). In the mid- to high-latitude regions large-scale precipitation contributes towards a positive heating. Diabatic cooling, on the other hand, is mostly caused by outgoing longwave radiation and, to a moderate extent, by the conversion process from rain to snow, mostly in the subtropical regions.

We see an extension of the area of positive heating in the midlatitudes towards the poles in the lower troposphere as well as in the equatorial mid- and upper troposphere for larger values of $\mathrm{OHT}_{\max }$. The poleward migration of the positive heating pattern in midlatitudes is closely related to the poleward shift of the atmospheric latent heat transport. The area of positive heating broadens in height at latitudes around $50^{\circ}$. Since the positive heating patterns (relevant for defining $\Theta^{+}$) in midlatitudes extend in height and are, in addition, stretched poleward, lower temperatures are considered in evaluating $\Theta^{+}$, which explains the smaller sensitivity of $\Theta^{+}$than of $\Theta^{-}$for $0 \mathrm{PW} \leq \mathrm{OHT}_{\max } \leq 1.5 \mathrm{PW}$ in Fig. 5. By implication, the warming effect at polar latitudes causes the sensitivity of $\Theta^{-}$to be larger than that of $\Theta^{+}$. For $\mathrm{OHT}_{\max } \geq 2 \mathrm{PW}$ the sensitivity of both $\Theta^{+}$and $\Theta^{-}$ is negative since large parts of the tropical high- and midtroposphere cool down.

We observe, on average, a decline in $\Delta \Theta$ of approximately $0.4 \mathrm{~K}$ for every $0.5 \mathrm{PW}$ increase in $\mathrm{OHT}_{\max }$ (Fig. 7; black 

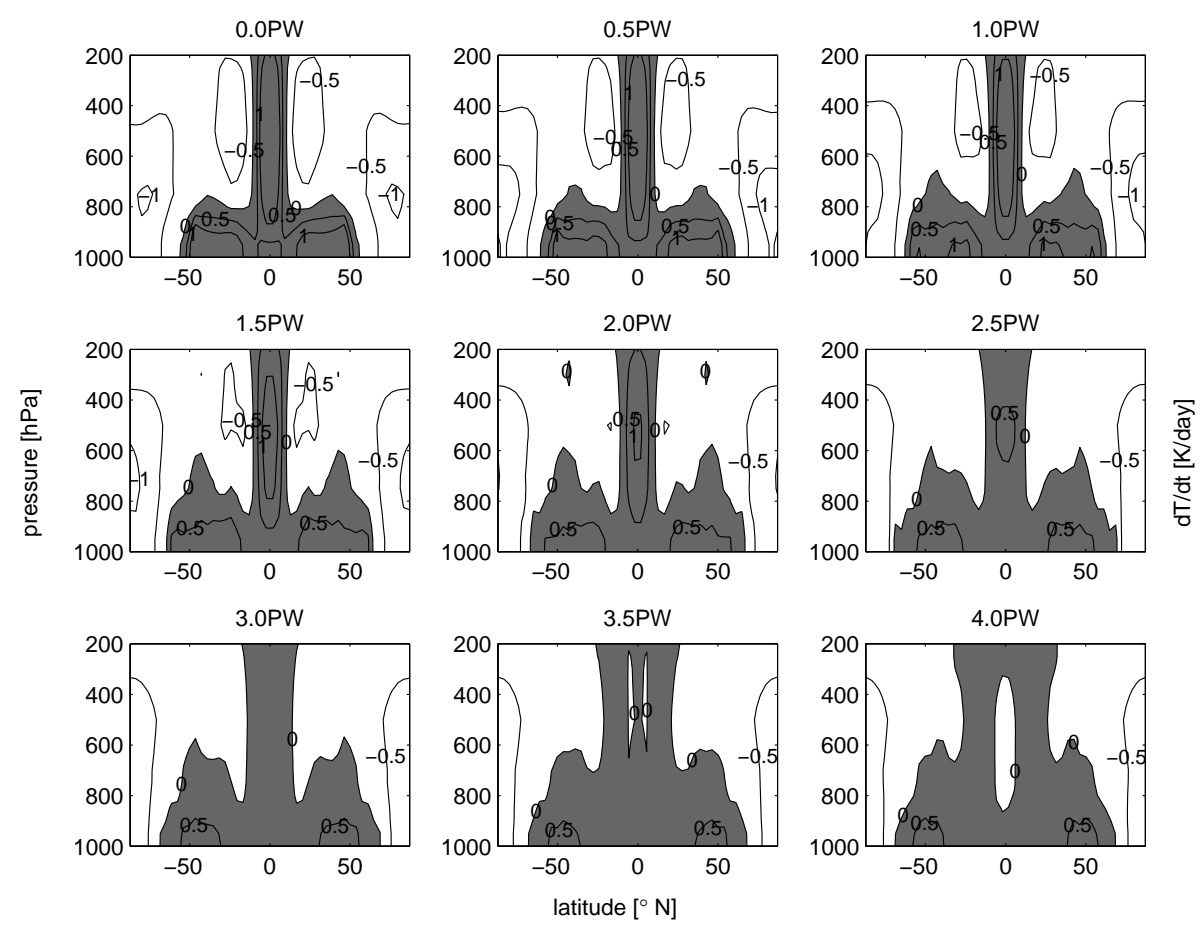

Figure 6. Zonally averaged mean heating rates in the atmosphere for oceanic heat transport ranging from $0.0 \mathrm{PW}$ (top left panel) to $4.0 \mathrm{PW}$ (bottom right panel), where grey-shaded areas indicate positive and white areas negative heating rates in $\mathrm{Kday}^{-1}$.

graph). The temperature difference $\Delta \Theta$ decreases from 7.9 to $4.5 \mathrm{~K}$ across the considered range of values of $\mathrm{OHT}_{\max }$. The climate system becomes horizontally more isothermal as $\mathrm{OHT}_{\max }$ is reinforced, which is consistent with the decline for the meridional difference in near-surface temperature $\Delta T$ (Fig. 7 blue graph, and Fig. 2). We find a linear relation between $\Delta T$ and $\Delta \Theta$ : for every $10 \mathrm{~K}$ decline in $\Delta T$, the reservoir temperature difference $\Delta \Theta$ decreases by approximately $0.8 \mathrm{~K}$ (Fig. 7). This provides a potentially interesting indication of how to relate changes in the near-surface temperature gradient to quantities describing the dynamic processes in the atmosphere.

As the climate warms and the temperature difference between the warm and the cold reservoir shrinks with increased $\mathrm{OHT}_{\max }$, the efficiency $\eta$ declines (Fig. 8). The increase in $\mathrm{OHT}_{\max }$ causes the climatic machine to act less efficiently, in terms of a decrease in the ratio between mechanical energy output and thermal energy input.

We observe a linear behavior for $\eta$ for $0 \mathrm{PW} \leq \mathrm{OHT}_{\max } \leq 2.5 \mathrm{PW}$. For every $0.5 \mathrm{PW}$ increase in $\mathrm{OHT}_{\text {max }}$ the efficiency, $\eta$ declines by about $2.0 \times 10^{-3}$. For $\mathrm{OHT}_{\max }$ larger than present-day values $\left(\mathrm{OHT}_{\max } \geq 2.5 \mathrm{PW}\right)$, $\eta$ decreases by only $0.5 \times 10^{-3}$ per $0.5 \mathrm{PW}$ increase. We observe an abrupt change in the tendency for $\mathrm{OHT}_{\max }=2.5 \mathrm{PW}$, at which pronounced tropical and subtropical atmospheric cooling sets in. This indicates that the change in the temperature difference between equatorial and tropical regions cause a drastic change in the dynamical properties of the system.

\subsubsection{Entropy budget}

We complete our analysis of the thermodynamics of the system by looking into how changes in the meridional oceanic heat transport impact the entropy budget.

As introduced in Appendix B, material entropy production $\overline{\dot{S}}_{\text {mat }}$ is given by the sum of the minimum value of entropy production $\left(\overline{\dot{S}_{\min }}\right)$ compatible with the presence of the average dissipation and the excess of entropy production $\left(\overline{\dot{S}_{\text {exc }}}\right)$ with respect to such a minimum, i.e.,

$\overline{\dot{S}_{\text {mat }}}=\overline{\dot{S}_{\text {min }}}+\overline{\dot{S}_{\text {exc }}}$.

The ratio between $\overline{\dot{S}_{\text {exc }}}$ and $\overline{\dot{S}_{\text {min }}}$ defines the degree of irreversibility $\alpha$,

$\alpha=\frac{\overline{\dot{S}_{\mathrm{exc}}}}{\overline{\dot{S}_{\text {min }}}}$,

and determines the ratio between the contributions to entropy production by down-gradient turbulent transport and by viscous dissipation of mechanical energy. Material entropy production $\overline{\dot{S}_{\text {mat }}}$ and the degree of irreversibility $\alpha$ are shown in Fig. 9 (upper panel). 

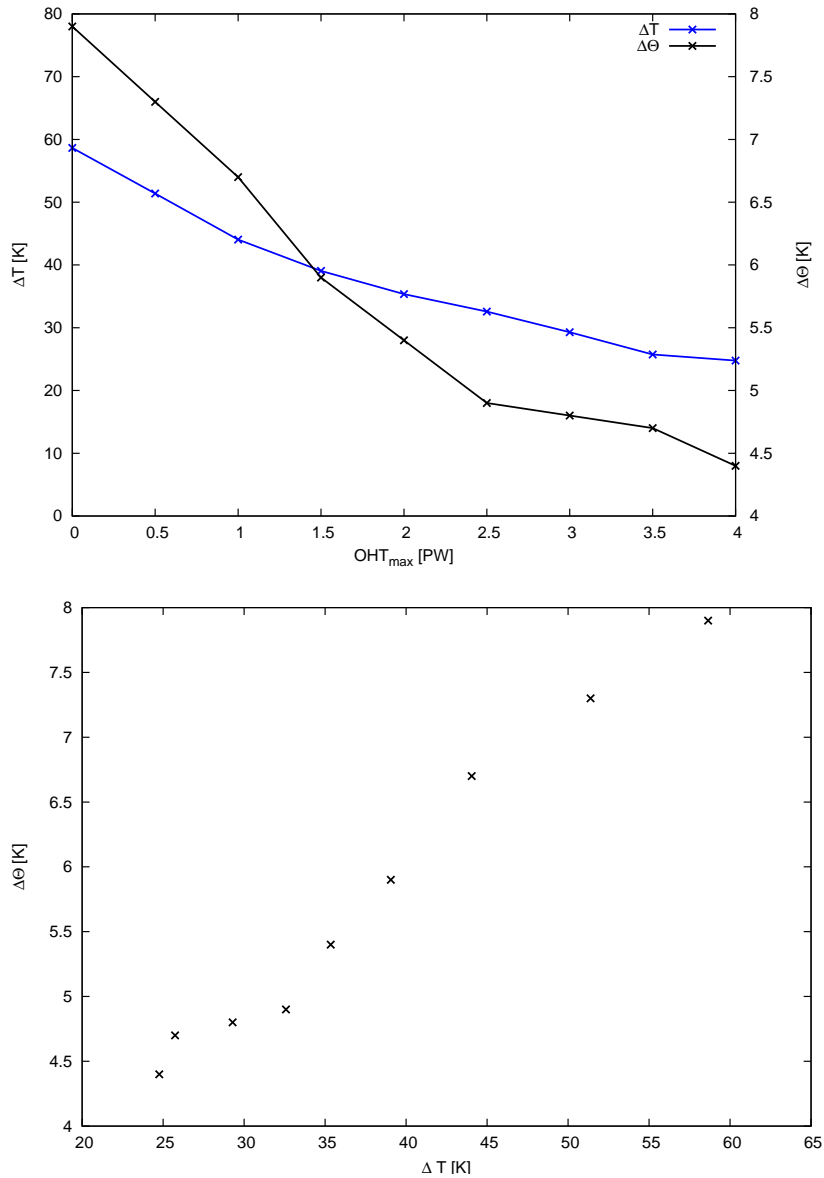

Figure 7. Upper panel: scatterplot of time-averaged global mean near-surface temperature difference between equator and pole (blue) as well as $\Theta^{+}$and $\Theta^{-}$(green) as a function of maximum energy transport in the ocean. Lower panel: scatterplot of timeaveraged global mean temperature difference between equator and pole as well as $\Theta^{+}$and $\Theta^{-}$.

With increasing values of $\mathrm{OHT}_{\max }$, the decrease in the efficiency (i.e., the intensity of the Lorenz energy cycle) and the increase in the near-surface temperature imply a reduction in the part in $\overline{\dot{S}_{\text {mat }}}$ linked with frictional dissipation, which is related to the lower bound of entropy production

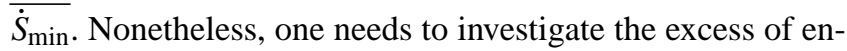
tropy production $\overline{\dot{S}_{\text {exc }}}$, which is linked to the turbulent heat fluxes down the temperature gradient. The relative decrease in entropy production due to frictional dissipation $\left(\overline{\dot{S}}_{\min }\right)$ is stronger than the relative decrease in entropy production by down-gradient turbulent heat transport $\left(\overline{\dot{S}}_{\text {exc }}\right)$ as featured by the overall increase in $\alpha$ (Fig. 9). Thus, the entropy production due to the turbulent heat transport down the gradient of the temperature field becomes more and more dominant as the oceanic transport increases because irreversible mixing becomes stronger.

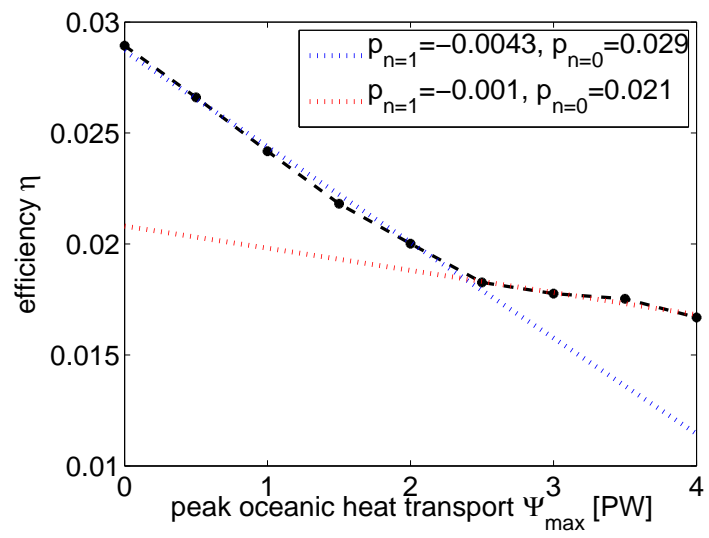

Figure 8. Time average of efficiency $\eta$ for steady state obtained for varying oceanic heat transport. Dotted line represents best linear fit for (i) $0.0 \mathrm{PW} \leq \mathrm{OHT}_{\max } \leq 2.5 \mathrm{PW}$ (blue) and for (ii) $2.5 \mathrm{PW} \leq \mathrm{OHT}_{\max } \leq 4.0 \mathrm{PW}$ (red) with polynomial coefficients of $n$th order, $p_{n=1}$ and $p_{n=0}$.
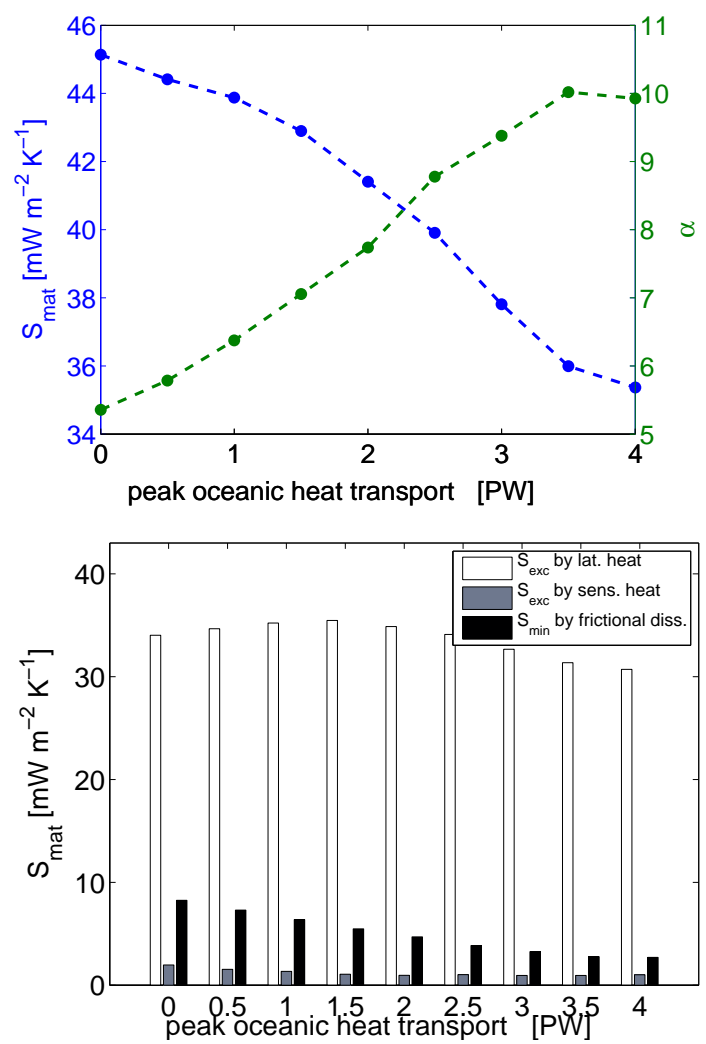

Figure 9. Upper panel: steady-state global mean material entropy production $\overline{\dot{S}}_{\text {mat }}$ (blue graph) and degree of irreversibility $\alpha$. Lower panel: most relevant contributions of $\overline{\dot{S}_{\text {mat }}}$ split into $\overline{\dot{S}_{\text {exc }}}$ and $\overline{\dot{S}_{\text {min }}}$, as a function of increasing oceanic heat transport.

In Fig. 9 (lower panels) the main contributions to the material entropy production in the model are displayed. This includes latent and sensible turbulent heat fluxes and 


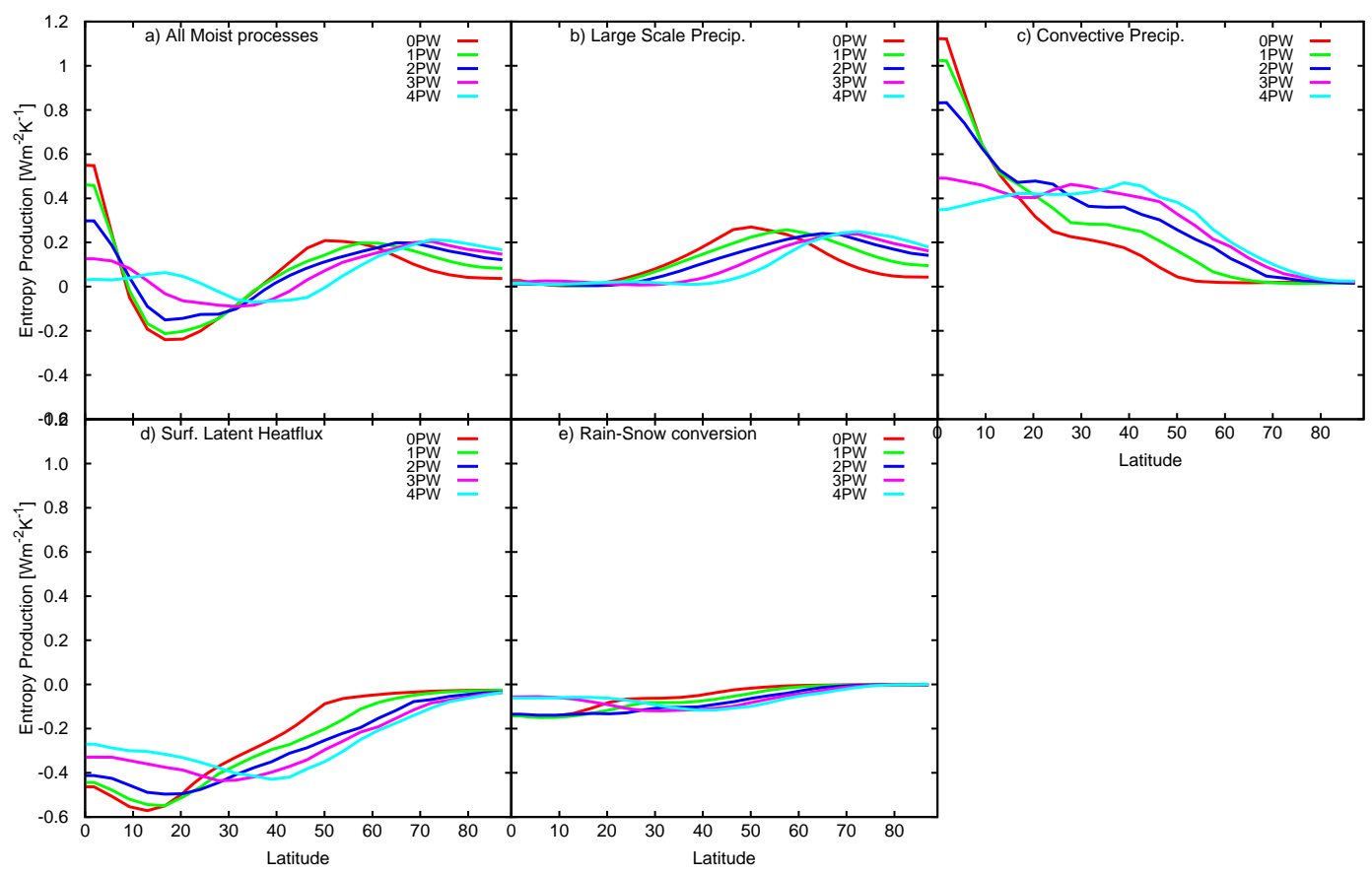

Figure 10. Entropy production from moist processes for $\mathrm{OHT}_{\max }=0,1,2,3$, and $4 \mathrm{PW}$.

frictional dissipation of kinetic energy. Entropy production due to latent heat processes, including convective as well as large-scale precipitation, surface latent heat fluxes and rain-snow conversion processes, makes up by far the largest portion of material entropy production. For small $\mathrm{OHT}_{\max }$, the value of entropy production by latent heat is $35 \mathrm{~mW} \mathrm{~m}^{-2} \mathrm{~K}^{-1}$. One would expect that larger values of OHT $_{\text {max }}$ would lead to larger values of $\overline{\dot{S}}_{\text {mat }}$, using the argument that a warmer planet should be able to have a stronger hydrological cycle, but things are in fact more complicated. For increasing $\mathrm{OHT}_{\max }$ up to $1.5 \mathrm{PW}$, the value increases by $2 \mathrm{~mW} \mathrm{~m}^{-2} \mathrm{~K}^{-1}$, while for larger $\mathrm{OHT}_{\max }$, this contribution to entropy production declines by $4 \mathrm{~mW} \mathrm{~m}^{-2} \mathrm{~K}^{-1}$. Entropy production by frictional dissipation decreases from $8 \mathrm{~mW} \mathrm{~m}^{-2} \mathrm{~K}^{-1}$ for $\mathrm{OHT}_{\max }=0 \mathrm{PW}$ to $3 \mathrm{~mW} \mathrm{~m}^{-2} \mathrm{~K}^{-1}$ for $\mathrm{OHT}_{\max }=4 \mathrm{PW}$. Entropy production by sensible turbulent heat flux at the surface as well as in the atmosphere decreases by half (from 2 to $1 \mathrm{~mW} \mathrm{~m}^{-2} \mathrm{~K}^{-1}$ ) with $\mathrm{OHT}_{\max }$ increasing. For low values of $\mathrm{OHT}_{\max }$, the increase in $\overline{\dot{S}}_{\text {mat }}$ due to the hydrological cycle is overcompensated for by the decrease in the contribution due to the frictional dissipation.

In order to further clarify the impact on the material entropy production of increasing $\mathrm{OHT}_{\max }$, we split the material entropy production due to irreversible latent turbulent heat processes ( $\boldsymbol{F}_{\text {lat }}$ in Eq. B6) into the contributions coming from individual parameterizations (processes) operating in our model, which are convective precipitation, large-scale precipitation, surface latent heat fluxes, and the heat release due to rain-snow conversion. Figure 10 displays the time mean of these contributions coming from each latitudinal belt. Positive contributions correspond to net warming, while negative contributions are related to cooling.

Convective precipitation gives the largest positive contribution, particularly in the tropics and subtropics. For increased $\mathrm{OHT}_{\max }$, we observe that the peak at the equator is significantly reduced while convection processes move into the midlatitudes where the surface is heated and static stability decreases. The positive contribution from large-scale precipitation features are shifted out of the midlatitudes towards higher latitudes with increasing $\mathrm{OHT}_{\max }$. As large-scale precipitation regimes experience a shift to higher latitudes, their maximum intensity is almost kept constant. The contribution by the surface latent heat flux is negative and related to surface cooling. For $0 \mathrm{PW}$, the largest values are obtained at latitudes of 20 to $25^{\circ}$, indicating the region with maximum evaporation. As the heat transport in the ocean is increased, latent turbulent heat fluxes are largely reduced in tropical and subtropical regions, and the maximum of latent heat fluxes move towards midlatitudes. The region with the largest evaporation at the surface shifts from the subtropics to the midlatitudes with increasing $\mathrm{OHT}_{\max }$. The contribution from latent heat release by rain-snow conversion is negative (indicating an overall cooling) and shows qualitatively similar patterns to the meridional profile of convective processes.

Figure 10 seems to imply that the tropical latitudinal belt features a negative material entropy production. This is indeed not the case because there is a net large-scale transport of energy from those regions to both the equator and the midlatitudes as a result of a net moisture export (with the 


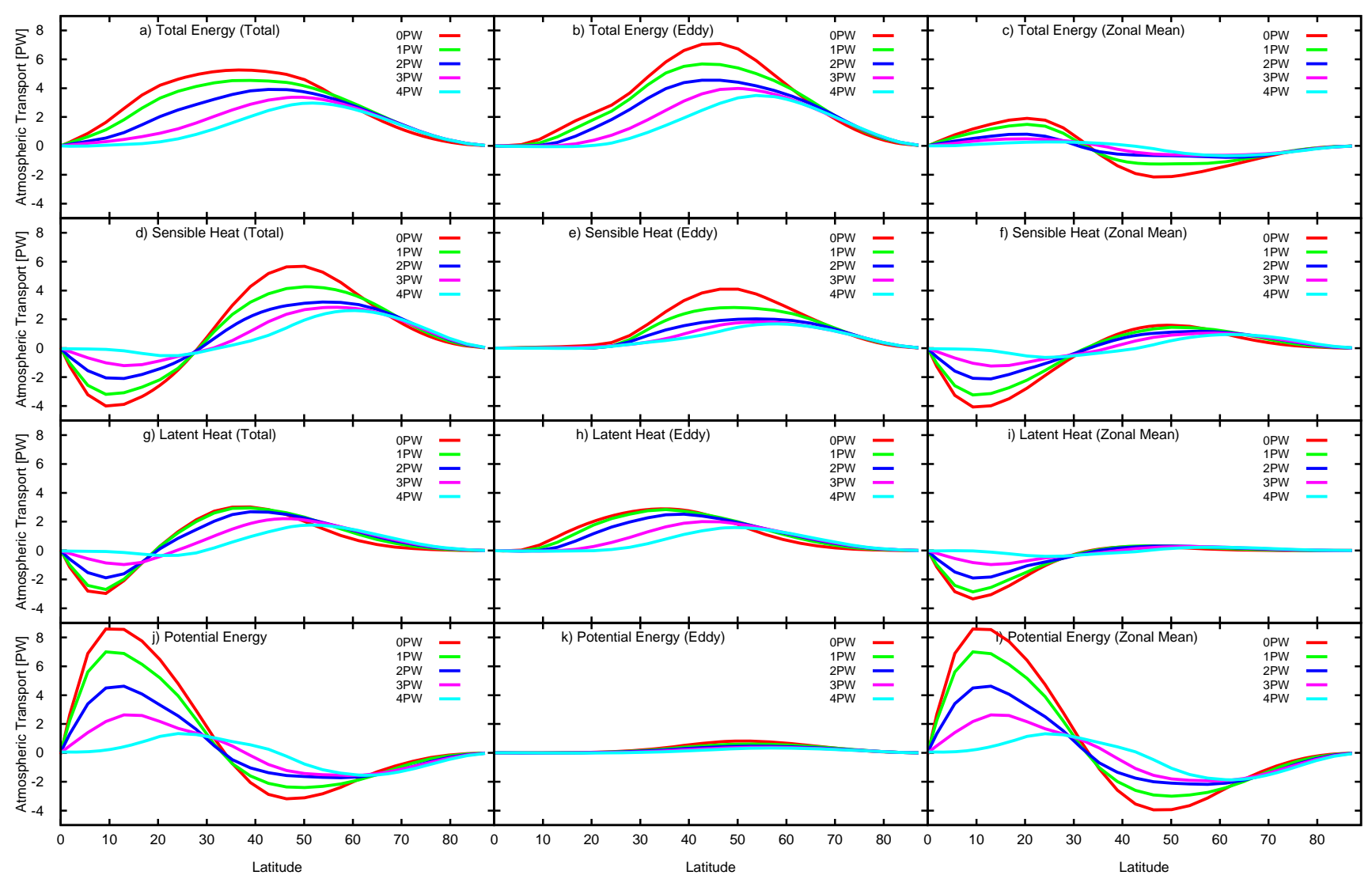

Figure 11. Atmospheric heat (moist static energy) transport (in PW) assigned to different processes for $\mathrm{OHT}_{\max }=0,1,2,3$, and 4 PW.

corresponding transport of latent heat; see discussion below). Such a negative contribution is overcompensated for by the positive material entropy production associated with the absorption of the transported latent heat taking place elsewhere. In addition, we note that the way we compute the entropy production associated with the hydrological cycle relies on focusing on water phase changes and related latent heat release and/or absorption; see Eqs. (B5)-(B6). Using a moist entropy that is mostly conserved in pseudoadiabatic motions would lead to a different partitioning of the material entropy production between precipitation, surface latent heat flux and rain-snow conversion.

\subsection{Atmospheric circulation and transports}

Now we discuss the sensitivity of the atmospheric circulation and transports to changes in OHT. Figure 11 shows the annual mean atmospheric meridional heat transport for $\mathrm{OHT}_{\max }=0,1,2,3$ and $4 \mathrm{PW}$. We present the total transport and its components: the transport of sensible heat, latent heat and potential energy. In addition, we split each transport into the contribution from the zonally symmetric (zonal mean flow) and the asymmetric (eddy) part. For the total transport, the compensation for increasing OHT leads to a decrease in the atmospheric transport and a poleward shift of its max- imum according to the prescribed OHT profile. Although the OHT is zonally symmetric, both atmospheric zonal mean flow and atmospheric eddies contribute to the compensation.

In the tropics $\left(0-30^{\circ}\right)$, both the zonal mean flow and the eddies account for the atmospheric transport, with the eddy component being dominant in the outer tropics, where the zonal mean flow contribution decreases to 0 . For the eddy transport in the tropics, only the latent heat transport is of appreciable magnitude. For the zonal mean flow, the magnitude of all three components decreases with increasing OHT showing about the same relative change per $1 \mathrm{PW} \mathrm{OHT}_{\max }$. The total mean flow transport is the result of a large compensation of the equatorward transport of heat (sensible and latent) and the poleward transport of potential energy.

In midlatitudes, eddies dominate the poleward heat transport and its sensitivity to OHT changes, with the contribution from latent heat transport being concentrated equatorward of the contribution from sensible heat transport. Transport of potential energy by eddies is almost absent due to their geostrophic nature (i.e., the meridional velocity is given by the zonal gradient of the geopotential, and thus the zonal average of the product of velocity and geopotential vanishes).

In summary, the atmospheric compensation for changes in OHT takes place according to the relative importance of the 

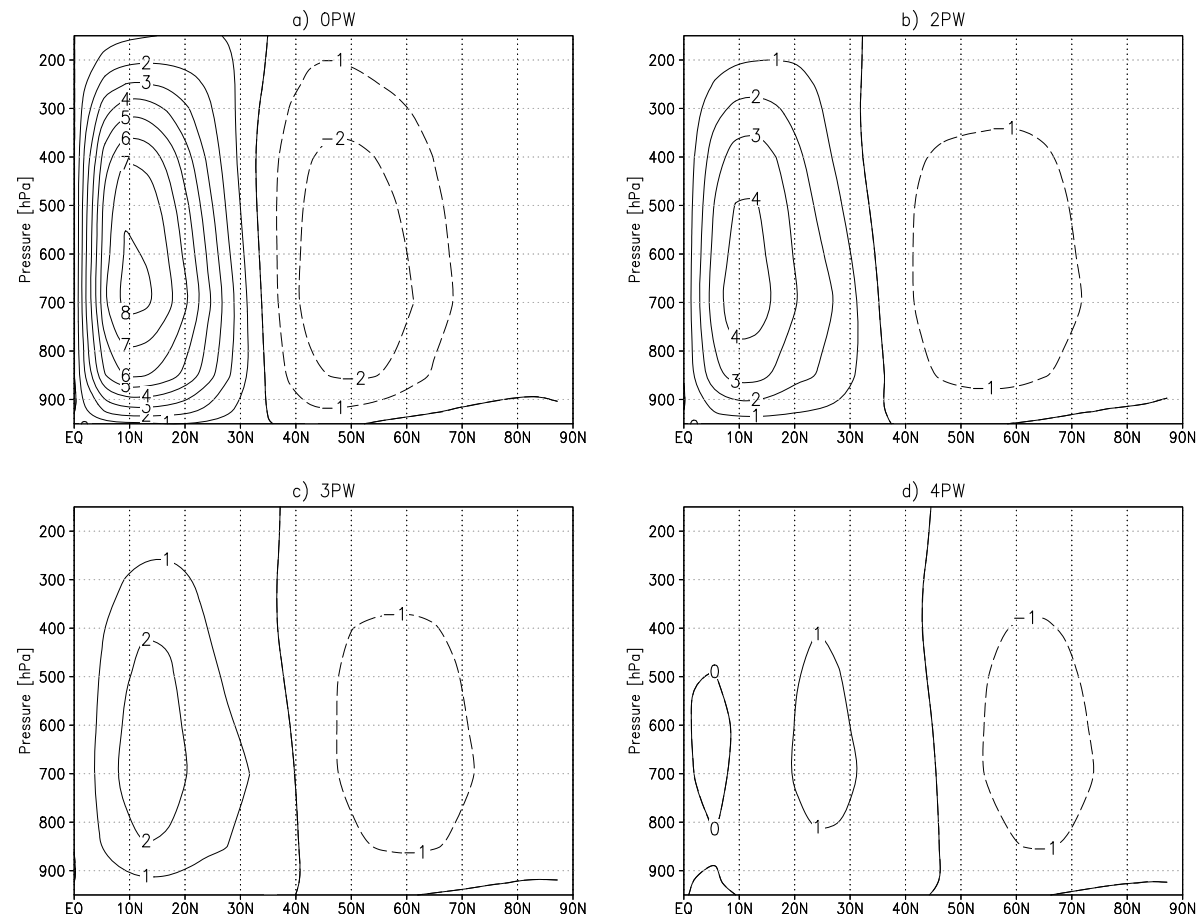

Figure 12. Climatological annual mean mass stream function (in $10^{10} \mathrm{~kg} \mathrm{~s}^{-1}$ ) for $\mathrm{OHT}_{\max }=0,2,3$, and $4 \mathrm{PW}$.

respective component for the transport in the reference state where no OHT is present. Even if changes in OHT are very large, it appears that the role of the different mechanisms in controlling the total heat transport remains unchanged: in the inner tropics eddy transport is not important and the poleward energy transport is due to the transport of potential energy by the zonal mean flow. Here, the transport of sensible and latent heat by the zonal mean flow is directed towards the equator, reducing the net transport. Poleward of the outer tropics the eddy transport becomes dominant. The importance of eddy latent transport increases for increasing temperatures as the moisture content is broadly controlled by the Clausius-Clapeyron law, so that the latent heat transport is more important for lower latitudes. Eddy transport of potential energy is negligible, while the transport of potential energy by the zonal mean flow in the midlatitudes is equatorward and counteracts the eddy transport.

The meridional atmospheric energy transport is closely linked to the mean meridional circulation, which we will study in the following. We start with the classical Eulerian mean circulation described by a mass stream function $\psi$. Figure 12 shows the Northern Hemisphere $\psi$ for $\mathrm{OHT}_{\max }=0$, 2, 3 and 4 PW. For $\mathrm{OHT}_{\max }=0 \mathrm{PW}$, a Hadley cell and a Ferrel cell are well established, with values of about $8 \times 10^{10}$ and $-3 \times 10^{10} \mathrm{~kg} \mathrm{~s}^{-1}$, respectively. The maximum magnitudes are located at about $10^{\circ} \mathrm{N}$ for the Hadley cell and $50^{\circ} \mathrm{N}$ for the Ferrel cell and at about $700 \mathrm{hPa}$ for both cells. The Hadley cell extends to about $33^{\circ} \mathrm{N}$. A polar cell is absent in the annual mean but emerges weakly in the summer months.

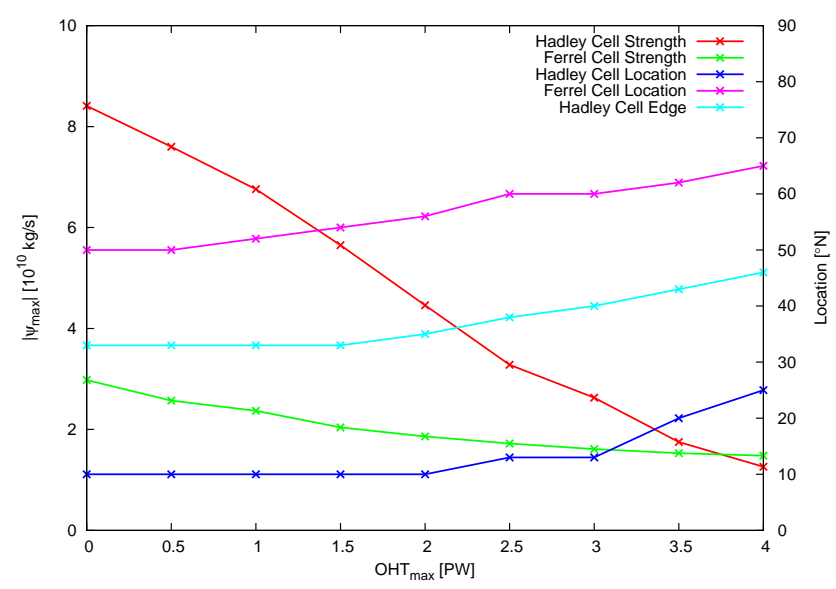

Figure 13. Climatological annual mean mass stream function (Northern Hemisphere): strength (in $10^{10} \mathrm{~kg} \mathrm{~s}^{-1}$ ) and location (in ${ }^{\circ} \mathrm{N}$ ) of Hadley and Ferrel cell for all simulations.

Considering the idealized setup, both the position and the strengths of the simulated cells are in reasonable agreement with observations for $\mathrm{OHT}_{\max }=2 \mathrm{PW}$, which is about the observed OHT strength (e.g., Peixoto and Oort, 1992).

With increasing OHT, the strength of both cells decreases (Fig. 13). The decrease in strength of the Hadley cell is virtually linear and amounts to about $1.8 \times 10^{10} \mathrm{~kg} \mathrm{~s}^{-1}$ per PW. The Ferrel cell strength decreases by about $0.4 \times 10^{10} \mathrm{~kg} \mathrm{~s}^{-1}$ per PW, with stronger decreases for smaller $\mathrm{OHT}_{\max }$. The 
core of the Ferrel cell shifts poleward. For $\mathrm{OHT}_{\max }>2 \mathrm{PW}$, a poleward shift can also be observed for the Hadley cell's maximum together with a broadening of this cell, i.e., a poleward shift of its edge. For $\mathrm{OHT}_{\max }=4 \mathrm{PW}$, an additional (weak) cell can be observed close to the equator with counterclockwise rotation. However, this (virtual) cell is caused by averaging an almost vanished Hadley cell in summer and a winter hemisphere Hadley cell which has its maximum in the summer hemisphere.

The Kuo-Eliassen equation allows for identifying individual drivers of the Eulerian mean meridional circulation (Appendix A). The reconstructions by means of the KuoEliassen equation are in good agreement with the actual $\psi$ for all simulations. However, the maximum magnitudes are systematically overestimated. In general, the reconstruction is a better fit for the Hadley cell than for the Ferrel cell (Fig. 15). It is not clear why the reconstruction overestimates the magnitudes of the cells. Possible sources of the differences are the numerical procedure to solve the equation (e.g., the representation of the derivatives) and, in particular, the quasi-geostrophic assumption.

As an example, Fig. 14 shows the sources and the reconstruction for $\mathrm{OHT}_{\max }=0 \mathrm{PW}$. The individual sources indicate that the largest contributions to $\psi$ stem from diabatic heating and from friction. The heating controls the Hadley cell together with a significant contribution by friction. For the Ferrel cell, friction is the most important factor. Eddy transports of heat and momentum add less to the Ferrel circulation. For both Hadley and Ferrel cell, the maximum contribution by friction is located at lower levels than for all other sources, which indicates the dominance of frictional dissipation close to the surface.

For the Hadley cell both the contributions coming from heating and friction decrease linearly with increasing OHT (Fig. 15). As the decrease is stronger for heating, friction becomes the major contributors to the Hadley cell for $\mathrm{OHT}_{\max }>3 \mathrm{PW}$. The decrease in the Ferrel cell with increasing OHT is linked to a decrease in the friction, i.e., a decrease in the near-surface zonal mean zonal wind. The contributions from heat and momentum transports decrease less than for the Hadley cell and remain constant for $\mathrm{OHT}_{\max }>2 \mathrm{PW}$. Similar to changes in magnitude, the shifting of the cells and the broadening of the Hadley cell can be explained by respective changes in the mean sources.

The residual mean stream function $\psi_{\text {res }}$ resulting from the TEM formalism (Andrews and McIntyre, 1976) provides a much closer link between the meridional circulation and the atmospheric transport of dry static energy. In addition, it clarifies the role of the eddies for the transport.

As given in Appendix A, the TEM formulations results in a decomposition of $\psi_{\text {res }}$ similar to the Kuo-Eliassen equation. Here, the residual mean circulation is the part of the mean meridional circulation that is not balanced by the convergence of the eddy heat transport. It consists of the Eulerian mean circulation plus a circulation due to the eddy heat trans- port. The latter is sometimes referred to as the Stokes stream function. The TEM residual mean circulation is qualitatively similar to the dry isentropic circulation with the important difference that the TEM circulation does not close at the surface (Held and Schneider, 1999). The residual stream function is forced by the combined effect of the eddy momentum and the eddy heat transport, given by the divergence of the Eliassen-Palm (E-P) flux. Splitting the Eliassen-Palm flux into its components shows that the individual contribution of the momentum transport is the same for both the Eulerian mean and the TEM formulation. Also, the sources from diabatic heating and friction remain the same.

Figure 16 gives the residual mean stream function, its reconstruction, the eddy source resulting from E-P flux divergence, and the Stokes stream function for $\mathrm{OHT}_{\max }=0 \mathrm{PW}$. As for the Eulerian mean, the reconstruction is in good agreement with the actual circulation (this again holds for all OHTs). Compared to the Eulerian mean (Fig. 14), the residual stream function displays a single overturning circulation with rising motion in the tropics and sinking air in high latitudes. While the maximum of the residual stream function occurs in midlatitudes near the surface, a secondary maximum in the tropics is present related to the Eulerian mean Hadley cell. From the reconstruction we see the dominance of the eddy (E-P flux) forcing for the midlatitudes compared to the Eulerian mean case. Since the contribution from the eddy momentum transport is the same in the Eulerian mean and the TEM case, the differences are due to the heat transport only. We also note that the Stokes stream function and E-P flux source are very similar, which is explained by the small contribution of the eddy forcing in the Eulerian mean case (note that the Stokes stream function is given by the difference between E-P flux source and Eulerian mean eddy source; cf. Appendix A).

Figure 17 displays the sensitivity of the E-P flux source and the Stokes stream function to changes in OHT. A strong decrease (from about $20 \times 10^{10} \mathrm{~kg} \mathrm{~s}^{-1}$ to about $9 \times 10^{10} \mathrm{~kg} \mathrm{~s}^{-1}$ ) of the maxima can be found for $\mathrm{OHT}_{\max }$ increasing from 0 to $2 \mathrm{PW}$. For $\mathrm{OHT}_{\max }>2 \mathrm{PW}$ the decrease is less steep and the maxima reach values of about $6 \times 10^{10} \mathrm{~kg} \mathrm{~s}^{-1}$. We note that, in the Eulerian mean view (Fig. 15), the eddy forcing does not appear to be very important because it is of small magnitude and has a limited sensitivity. The huge impact of the eddies on the circulation only becomes clear when considering the combined effect of the eddies, which sets up an eddy-related (Stokes) circulation, as visible in the TEM view.

Concerning the total heat transport, including latent heat, Czaja and Marshall (2006), based on work by Held (2001), showed that the atmospheric heat transport can be represented by the product of a moist TEM residual circulation and the vertical contrast in moist static energy (or equivalent potential temperature, $\theta_{\mathrm{e}}$ ). Here, both the eddy transport and the vertical gradient of $\theta$ in the TEM formalism are replaced by the respective values utilizing $\theta_{\mathrm{e}}$. Unfortunately, 
a) OPW Original

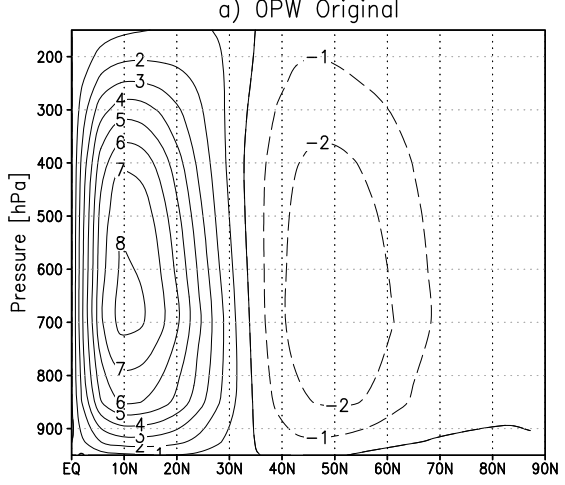

c) OPW Diabatic Heating

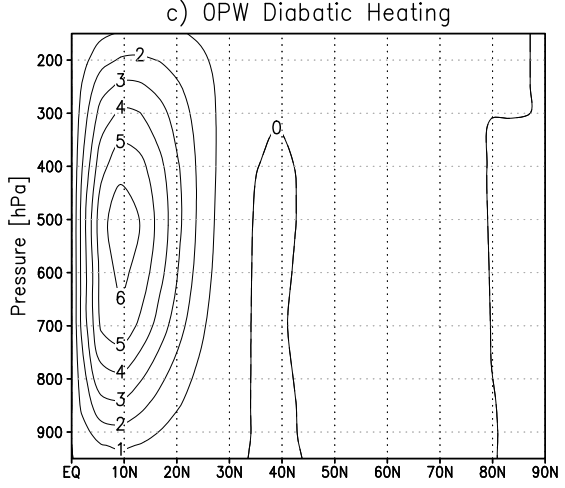

e) OPW Eddy Heat Transport

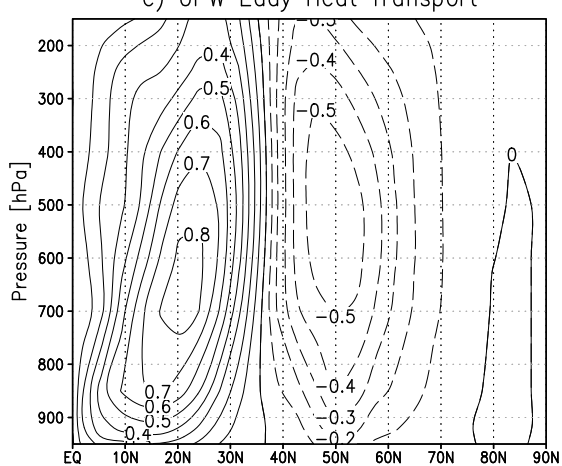

b) OPW Reconstructed

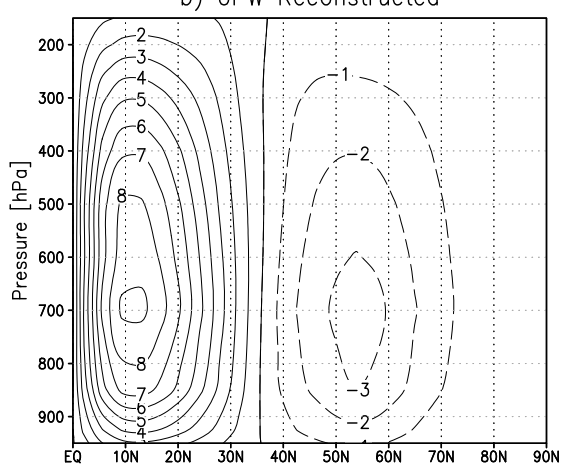

d) OPW Friction

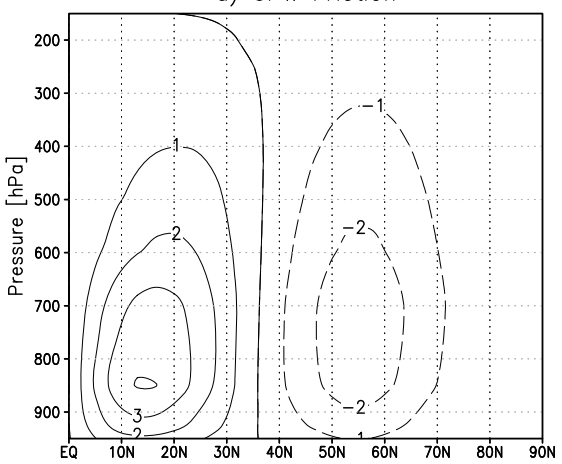

f) OPW Eddy Momentum Transport

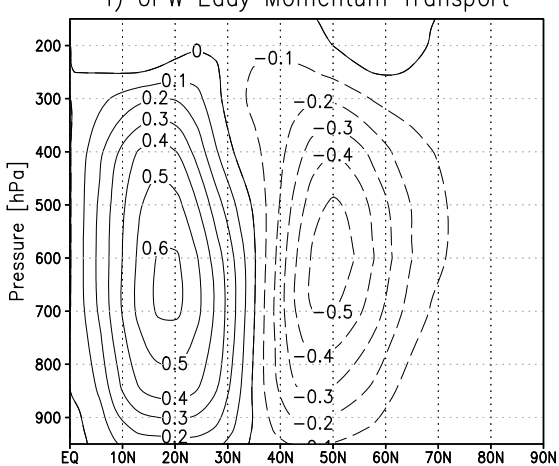

Figure 14. Climatological annual mean mass stream function (in $10^{10} \mathrm{~kg} \mathrm{~s}^{-1}$ ) for $\mathrm{OHT}_{\max }=0 \mathrm{PW}$ : (a) original (see Fig. 12); (b) computed from the Kuo-Eliassen equation (all sources); (c) source from diabatic heating; (d) source from friction; (e) source from eddy heat transport; (f) source from eddy momentum transport.

as mentioned in the introduction, there is no simple way to represent a well-defined moist isentropic circulation in the latitude-pressure plane (Pauluis et al., 2011; Laliberté et al., 2012). This prevents a diagnostic similar to the dry case. To tackle this problem, Pauluis et al. (2011) introduced a statistical generalization of the transformed Eulerian mean circulation for arbitrary vertical coordinates. However, here we restrict ourselves by diagnosing and comparing the total atmospheric circulation on dry and moist isentropes.

Figure 18 displays the respective circulations for $\mathrm{OHT}_{\max }=0$ and $3 \mathrm{PW}$, as well as the maxima of the respec- tive stream functions for different OHTs. The circulation on dry isentropes corresponds well with the residual circulation except that it is closed and has smaller maxima, mainly due to the misrepresentation of near-surface values in pressure coordinates. It shows one single overturning cell with (for small OHT) a tropical and a midlatitude maximum. In contrast to the dry case, the circulation on moist isentropes shows only one maximum for all values of OHT, which is located in the midlatitudes. In addition, the moist isentropic circulation is narrower and exhibits larger mass transport values, illustrating the impact of the moisture transport. 
a) Hadley Cell Sources

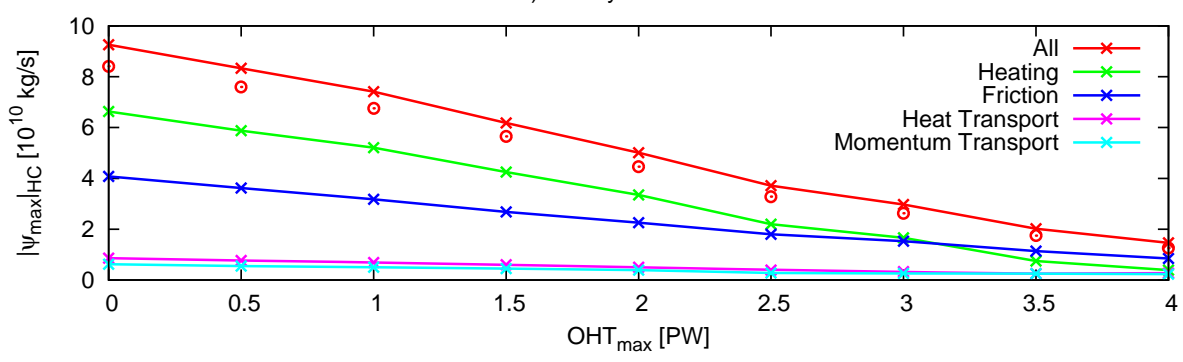

b) Ferrel cell Sources

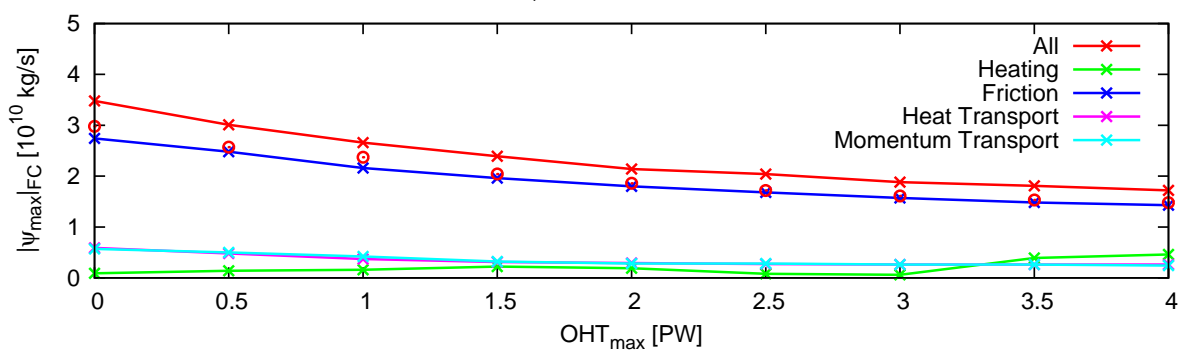

Figure 15. Sources (in $10^{10} \mathrm{~kg} \mathrm{~s}^{-1}$ ) of the Hadley (upper panel) and the Ferrel (lower panel) cell according to the Kuo-Eliassen equation. Circles indicate the actual strength of the respective cell.
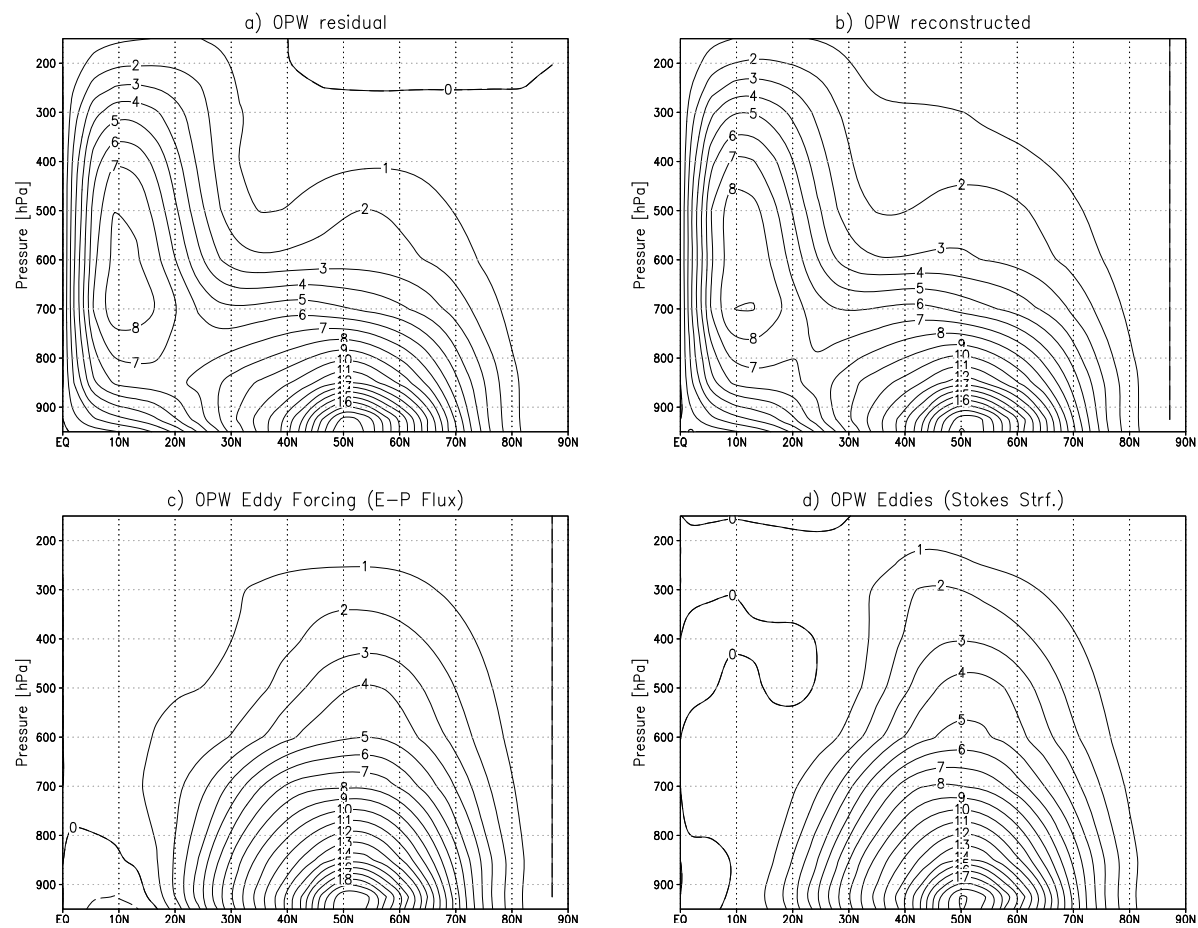

Figure 16. Climatological annual mean residual stream function (in $10^{10} \mathrm{~kg} \mathrm{~s}^{-1}$ ) for $\mathrm{OHT}_{\max }=0 \mathrm{PW}$ : (a) original; (b) reconstructed; (c) eddy source; (d) Stokes.

For increasing OHT, both the dry and the moist isentropic circulation slow down, and the maxima shift poleward. In accordance with Czaja and Marshall (2006), this agrees well with changes in the transport of dry and moist static energy (cf. Fig. 11). However, the relative decreases in the transports are smaller than those in the circulations. This is explained by a narrowing of the isentropic circulation for larger OHT 


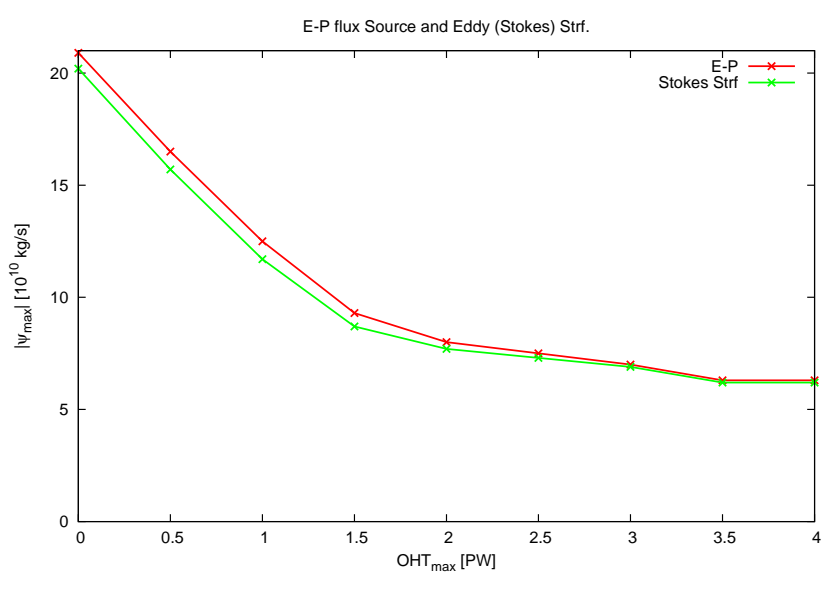

Figure 17. Eddy (E-P flux) source of the residual circulation and Stokes stream function (in $10^{10} \mathrm{~kg} \mathrm{~s}^{-1}$ ).

(cf. Fig. 18 for 0 and $3 \mathrm{PW}$ ) corresponding to a decrease in static stability.

\subsection{Lorenz energy cycle}

Finally, we give a synopsis of the above results in terms of the global energetics provided by the classical Lorenz energy cycle (Lorenz, 1955). In particular, this will confirm the relevance of the climate engine perspective.

Figure 19 shows reservoirs, conversions and sources of the energy cycle where the atmospheric flow has been partitioned into the zonal mean and the eddy component. We already noted (cf. Fig. 7) the close (linear) relation between $\Delta T$ (the meridional near-surface temperature gradient) and $\Delta \Theta$ (the temperature difference between the warm and the cold thermodynamic reservoirs of the climate engine). Consistent with the changes in $\Delta \Theta$ and $\Delta T$, the Lorenz available potential energies of the mean flow $\left(P_{\mathrm{M}}\right)$ and of the eddies $\left(P_{\mathrm{E}}\right)$ decrease. In addition, we notice that the relative decrease in $P_{\mathrm{M}}$ and $P_{\mathrm{E}}$ is of the same size, while the absolute values of $P_{\mathrm{M}}$ are substantially larger.

As pointed out in Appendix B, a direct link between the efficiency of the climate system $(\eta)$ and the strength of the Lorenz energy cycle is given by the work output $\overline{\dot{W}}$ of the climate engine, which gives the rate of generation of available potential energy. Indeed, for $\overline{\dot{W}}$ (Fig. 20) we observe a similar behavior as for $\eta$ (Fig. 8). For $0 \mathrm{PW} \leq \mathrm{OHT}_{\max } \leq 2.5 \mathrm{PW}, \overline{\dot{W}}$ decreases linearly by about $0.2 \mathrm{Wm}^{-2}$ for every $0.5 \mathrm{PW}$ increase in $\mathrm{OHT}_{\max }$. For $\left(\mathrm{OHT}_{\max } \geq 2.5 \mathrm{PW}\right), \overline{\dot{W}}$ declines by $0.1 \mathrm{Wm}^{-2}$ per $0.5 \mathrm{PW}$ increase.

From energy conservation we know that the decrease in $\overline{\dot{W}}$ also implies that the total dissipation $\overline{\dot{D}}$ decreases in a steadystate climate, as the climatic engine has a smaller rate of transformation of available into kinetic energy. The decrease in $\overline{\dot{D}}$ implies that surface winds are weaker because this is where most of the dissipation takes place. Though diagnosed as residuals from the conversions, the total source of available potential energy and the total sink of kinetic energy are in good agreement with $\overline{\dot{W}}$ and $\eta$, indicating a change in sensitivity at $\mathrm{OHT}_{\max }=2.5 \mathrm{PW}$. In addition, we notice that only the zonally symmetric heating generates available potential energy, while the zonally asymmetric heating extracts $P_{\mathrm{E}}$, i.e., it acts to homogenize the zonal temperature profiles. For the dissipation of kinetic energy, the eddy component is larger than the contribution of the zonal mean flow. For increasing OHT, the magnitude of all sources and/or sinks decrease and both the (negative) eddy source of available potential energy and the zonal mean sink of kinetic energy go to 0 .

We conclude that assigning the overall strength of the Lorenz energy cycle to either the zonal mean or the eddy flow would lead to different results depending on whether we choose the generation of available potential energy $\overline{\dot{W}}$ or the dissipation of kinetic energy $\overline{\dot{D}}$ as a measure. Dominant processes of the generation of available energy (i.e., $\overline{\dot{W}}$ ) are related to the zonal mean circulation, while the dissipation of kinetic energy $(\overline{\dot{D}})$ acts on the eddies resulting from baroclinic instability. In addition, one may use the conversion from potential to kinetic energy to define the energy cycle strengths. Here, the baroclinic eddies accomplish such a transformation, while the zonal mean flow generates $P_{M}$ at the expense of $K_{\mathrm{M}}$, i.e., by favoring a thermally indirect circulation. Thus, only considering both components together (eddy and mean flow) allows for assessing the strength of the energy cycle. In this respect, $\eta, \overline{\dot{W}}$ and $\overline{\dot{D}}$ derived from the (Carnot) climate engine perspective provide consistent and physically based measures.

Consistent with the sensitivity of the transports and the meridional circulation, the overall decline in the reservoirs and sources with increasing OHT is also present for conversion terms related to the baroclinic conversion, i.e., $C\left(P_{\mathrm{M}}\right.$, $\left.P_{\mathrm{E}}\right), C\left(P_{\mathrm{E}}, K_{\mathrm{E}}\right)$ and $C\left(K_{\mathrm{E}}, K_{\mathrm{M}}\right)$. The conversion $C\left(P_{\mathrm{M}}\right.$, $K_{\mathrm{M}}$ ), which is relevant for defining the zonal mean Eulerian circulation, shows little change, as there is a cancellation between the changes occurring in the Hadley and Ferrel cells. In addition, we note that the sensitivity of the eddy-related conversions appears to decrease following the temporal sequence of a baroclinic life cycle: the conversion from zonal mean available potential energy to eddy potential energy $C\left(P_{\mathrm{M}}, P_{\mathrm{E}}\right)$ shows the largest sensitivity (approx. $65 \%$ reduction for $4 \mathrm{PW}$ increase in OHT). The sensitivity of the transformation into eddy kinetic energy $C\left(P_{\mathrm{E}}, K_{\mathrm{E}}\right)$ amounts to approx. $57 \%$, and the change of the conversion into zonal mean kinetic energy $C\left(K_{\mathrm{E}}, K_{\mathrm{M}}\right)$ is the smallest (approx. $53 \%$ ). However, to verify whether these changes are due to changes in the baroclinic life cycles or just a coincidence, further analysis is necessary, which is beyond the scope of the present paper. 

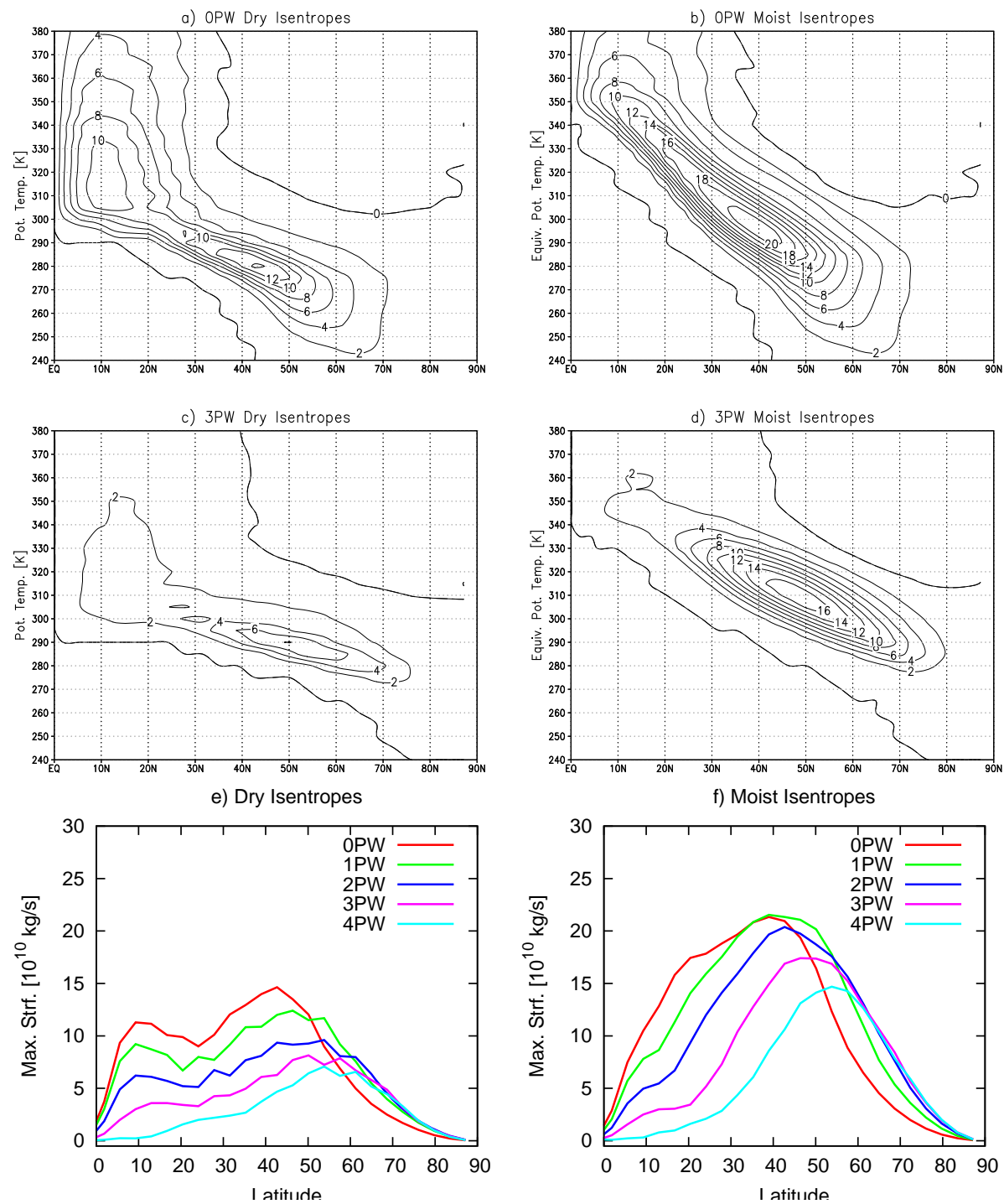

Figure 18. Upper panels: climatological annual mean mass stream function (in $10^{10} \mathrm{~kg} \mathrm{~s}^{-1}$ ) for $\mathrm{OHT}_{\max }=0 \mathrm{PW}$ on (a) dry isentropes and (b) moist isentropes. Middle panels: climatological annual mean mass stream function (in $10^{10} \mathrm{~kg} \mathrm{~s}^{-1}$ ) for $\mathrm{OHT}_{\max }=3 \mathrm{PW}$ on (c) dry isentropes and (d) moist isentropes. Lower panels: maximum of stream function (in $10^{10} \mathrm{~kg} \mathrm{~s}^{-1}$ ) on (e) dry isentropes and (f) moist isentropes for $\mathrm{OHT}_{\max }=0,1,2,3$, and $4 \mathrm{PW}$.

\section{Summary and discussion}

We have studied the impact of the oceanic heat transport (OHT) on the atmospheric circulation focusing on two important aspects: changes in the atmospheric meridional heat transport and circulation and changes in global thermodynamic properties of the atmosphere including efficiency, irreversibility and the Lorenz energy cycle.

Using a general circulation model of intermediate complexity (PlaSim) including an oceanic mixed layer, we have adopted an experimental design from Rose and Ferreira (2013). Here, an imposed oceanic heat transport of simple analytic form and with varying strengths allows for systematic analyses.
We found a compensation of the changes in oceanic heat transport by the atmosphere consistent with Stone's (1978) conclusions. The presence of sea ice may explain the deviations from a perfect compensation as discussed in Enderton and Marshall (2008). While all components of the atmospheric heat transport are affected by the compensation, their relative importance for the total transport remains almost unchanged. While the atmosphere counterbalances the changes in the OHT very effectively, so that the total meridional heat transport is weakly altered, the climate as a whole strongly depends on the OHT value chosen. The basic reason for this is that the atmosphere and the ocean transport heat at different heights and across different temperature gradients. 
a) Reservoirs

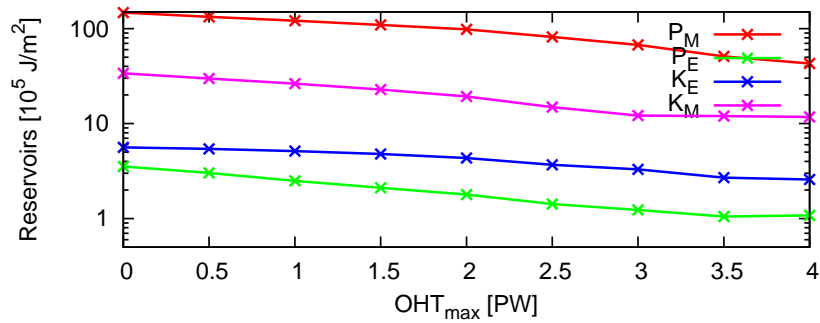

b) Conversions

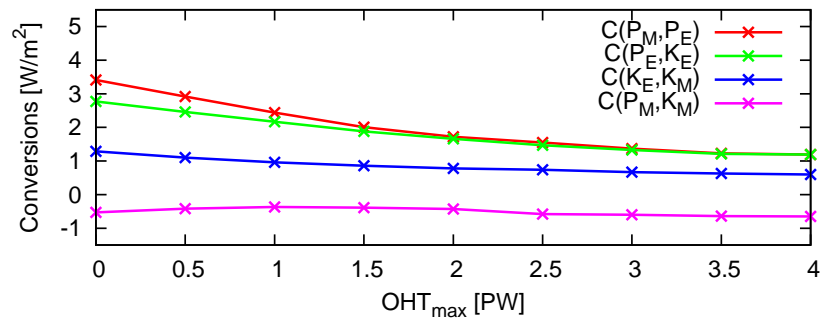

c) Sources

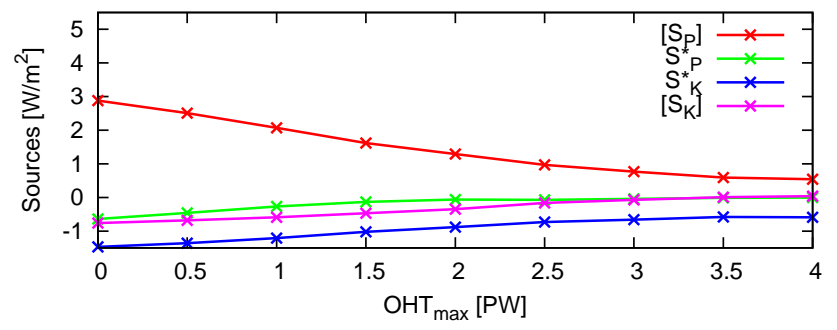

Figure 19. Climatological mean Lorenz energy cycle: reservoirs (upper panel, in $10^{5} \mathrm{~J} \mathrm{~m}^{-2}$ ), conversions (middle panel, in $\mathrm{W} \mathrm{m}^{-2}$ ) and sources (lower panel, in $\mathrm{W} \mathrm{m}^{-2}$ ) for all simulations.

In agreement with Rose and Ferreira, we have found an increase in the global mean near-surface temperature and a decrease in the equator-to-pole temperature gradient, with increasing OHT for $\mathrm{OHT}_{\max }<3 \mathrm{PW}$. For larger OHT, the temperature gradient still decreases but the global average remains constant. For the tropics, there is a significant decrease in both temperature and its gradient for $\mathrm{OHT}_{\max }>2 \mathrm{PW}$, with a reversal of the gradient for $\mathrm{OHT}_{\max }>3 \mathrm{PW}$. For smaller OHT, we observed a slight warming and a reduction in the gradient with increasing OHT. The latter is consistent with results from Koll and Abbot (2013). However, on their aquaplanet the tropical temperature showed little sensitivity, with small increases for all imposed (positive) OHTs (up to $3 \mathrm{PW}$ ).

A tropical cooling for imposed oceanic heat transports somewhat larger than present-day values has also been found by Barreiro et al. (2011) in a more complex coupled atmosphere-slab ocean model with a present-day land-sea distribution. They argue that this suggests that present-day climate is close to a state where the warming effect of OHT is maximized. Barreiro et al. (2011) related the tropical cool-

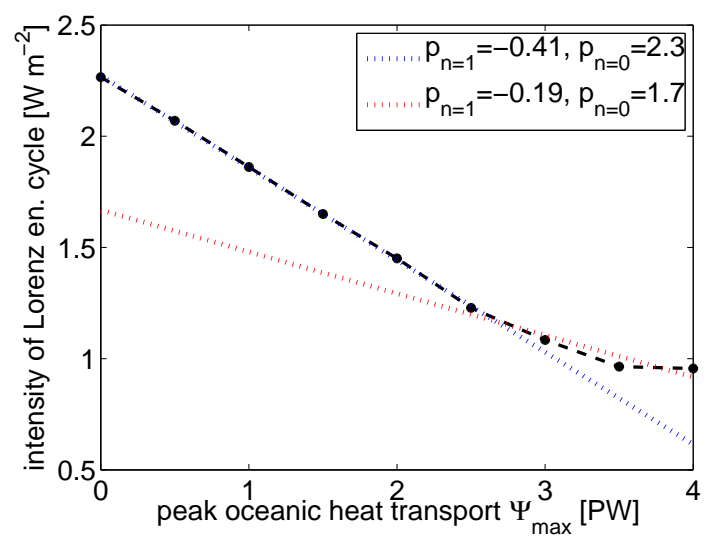

Figure 20. Time average of the intensity of the Lorenz energy cycle $\overline{\dot{W}}$ (lower) for steady state obtained for varying oceanic heat transport. Dotted line represents best linear fit for (i) $0.0 \mathrm{PW} \leq \mathrm{OHT}_{\max } \leq 2.5 \mathrm{PW}$ (blue) and for (ii) $2.5 \mathrm{PW} \leq \mathrm{OHT}_{\max } \leq 4.0 \mathrm{PW}$ (red) with polynomial coefficients of $n$th order, $p_{n=1}$ and $p_{n=0}$.

ing to a strong cloud-SST feedback and showed that the results are sensitive to the particular parameterizations. Though our simulations are highly idealized and do not represent all the complexities of the real climate system, it is interesting to note that we find almost no further increase in the global near-surface temperature for $\mathrm{OHT}_{\max }>2.5 \mathrm{PW}$ and maxima in $\Theta^{+}$and $\Theta^{-}$at about the same value of OHT.

Confirming the results of previous studies (Herweijer et al., 2005; Barreiro et al., 2011; Koll and Abbot, 2013), we have found a decrease in the Hadley cell for increasing OHT. In addition, the Hadley cell broadens and the maxima of the Hadley and the Ferrel cell shift poleward when OHT obtains large values $\left(\mathrm{OHT}_{\max }>2.5 \mathrm{PW}\right)$.

Sea ice gradually decreases with increasing OHT. Though in annual averages sea ice is present for all simulations, for $\mathrm{OHT}_{\max }>2 \mathrm{PW}$ areas of open water are present for all latitudes during summer. This may suggest that sea ice plays an important role in controlling the global mean temperature and/or the position of the Ferrel cell. However, we did not find sufficient evidence to support this hypothesis.

Separating individual sources by applying the KuoEliassen equation showed that the characteristics of the Hadley cell can be explained by the mean meridional circulations related to the diabatic heating and, to a smaller extent, to the friction. In our simulations, the meridional circulation induced by friction also controls the behavior of the Ferrel cell. Eddy transports of heat and momentum appear to be less important for the Eulerian mean circulation. This is different from results by Kim and Lee (2001b), where the mean meridional circulation related to eddy fluxes accounts for about $50 \%$ of the Ferrel cell's strength. The coarse vertical resolution may be responsible for a reduced eddy activity. 
The importance of the eddies for the circulation becomes clear when considering the combined effect of the eddies by applying the TEM formalism. Here, the eddies set up an eddy-related (Stokes) circulation dominating the midlatitudes with strong sensitivity to changes in OHT.

In agreement with Czaja and Marshall (2006) the total atmospheric meridional heat transport is related to the strength and vertical extent of the circulation on isentropes both for the dry and the moist case. As obtained by scale analysis by Czaja and Marshall, the residual mean stream function in the midlatitudes is dominated by the so-called eddy stress which is given by the meridional transport of static energy.

We utilized an alternative approach to assess the sensitivity of the climate system by studying the response of global thermodynamical properties of the climate system following a theoretical framework introduced by Lucarini (2009). Here, the climate system is viewed as an (equivalent Carnot) engine with a certain efficiency. Linking the climate engine view and the classical Lorenz energy cycle (Lorenz, 1955) provided a consistent picture of the observed changes and, thus, demonstrated the relevance of the climate engine approach.

Increasing OHT leads to a reduction in the difference between the warm pool temperature $\Theta^{+}$and the cold pool temperature $\Theta^{-}$. The latter implies that the atmospheric system becomes more isothermal in the horizontal. The temperature difference between the warm $\left(\Theta^{+}\right)$and the cold $\left(\Theta^{-}\right)$ heat reservoir decreases for increasing oceanic heat transport. This is basically caused by enhanced warming in the extratropics and by tropical cooling for increasing OHT. One of the main drivers for this is the poleward relocation of latent heat release patterns (not shown). This may lead to further warming due to the water vapor feedback (Herweijer et al., 2005; Barreiro et al., 2011).

The effect of thermalization leading to the reduction in the efficiency of the system with increasing intensity of the ocean heat transport can be related to the decrease in the reservoir of the potential energy available for conversion in the Lorenz energy cycle. The strength of the Lorenz energy cycle linearly decreases with increasing OHT. A change to smaller sensitivity is observed at $\mathrm{OHT}_{\max }=2.5 \mathrm{PW}$.

Consistent with the changes in heat transport and meridional circulation, the magnitude of all reservoirs and conversions of the Lorenz energy cycle decreases with increasing OHT. However, the sensitivities differ. $P_{\mathrm{M}}$ and the conversion from $P_{\mathrm{M}}$ to $P_{\mathrm{E}}$ exhibit the largest changes. Eddy kinetic energy, the barotropic conversion from eddy kinetic energy to zonal mean kinetic energy, and the conversion from zonal mean kinetic energy to $P_{\mathrm{M}}$ are least affected.

When considering stronger oceanic transport, the climate system is characterized by a declining total material entropy production, while the degree of irreversibility $\alpha$ increases, since the decrease in entropy production by frictional dissipation is more intense than the decrease in entropy generation due to sensible and, in particular, latent heat flux. The increase in the index of irreversibility is a direct consequence of the decrease in the efficiency $\eta$, due to the reduction in temperature gradients inside the system, in agreement with what is found in Lucarini et al. (2010a, b, 2013) and Boschi et al. (2013). The flux of latent heat contributes most to the material entropy production in the climate system. When increasing the heat transport in the ocean from 0.0 to $1.5 \mathrm{PW}$, material entropy production due to latent heat flux increases, which can be explained by an outspread of convection from the deep tropics into the midlatitudes, while the maximum latent release is still located in the central tropics. When increasing the heat transport further, convective processes collapse in the deep tropics and, thus, affecting evaporation intensities at tropical sea surface by reducing it. As a result, a decrease in material entropy production by latent heat fluxes can be noted from the increase in the oceanic heat transport larger than 2.0 PW.

Recently, Laliberté et al. (2015) proposed a different thermodynamic point of view from the one taken here, indeed confirming the relevance of looking at the climate system as a heat engine. They studied using models and reanalyzed the work output of the climate engine. In doing so, they showed that it is constrained by the power necessary to maintain the hydrological cycle, which accounts for the moisture inefficiency related to the addition of water vapor to unsaturated air. For a warmer climate they found a reduction in the work output consistent with our results for increasing OHT. Laliberté et al. (2015) attributed most of the response to an increase in the moistening inefficiency. There is a strong indication that this is also true in our case due to a large increase in near-surface specific humidity and evaporation with only moderate changes in near-surface relative humidity. However, further diagnostics are necessary to quantify the impact of moistening inefficiency.

Overall, our study demonstrates the large impact of the oceanic heat transport on the atmospheric circulation affecting the zonally symmetric flow, the zonally asymmetric flow and the interaction between both. By reducing the meridional temperature gradient, an increased oceanic heat transport slows down the atmospheric mean meridional circulation and shifts the Hadley and the Ferrel cell. In addition, changes in OHT substantially modify global thermodynamic properties such as the strength of the Lorenz energy cycle, the efficiency, the entropy production and the irreversibility.

The reduction in the meridional gradient of the nearsurface temperature is one of the major features of global warming. Lu et al. (2007) showed a consistent weakening and poleward expansion of the Hadley cell in IPCC AR4 simulations. Hence, changes in the oceanic heat transport may significantly modify the response of the atmospheric circulation to greenhouse warming. A weakening of the oceanic meridional overturning circulation, as predicted by the majority of coupled ocean-atmosphere general circulation models (though with large uncertainties; IPCC, 2013), would therefore act as a negative feedback mechanism. This negative feedback might become even more important when 
strong melting of inland ice sheets, due to global warming, is taken into account. The associated input of large amounts of freshwater has a huge potential to slow down the oceanic circulation.

Apart from the meridional overturning circulation in the Atlantic, significant modifications to the oceanic circulation in a warmer climate can also be found in the equatorial Pacific, and they are strongly linked to El Niño-Southern Oscillation (ENSO) variability (e.g., Collins et al., 2010; Fedorov et al., 2006). This gives rise to an additional potential feedback mechanism related to oceanic dynamics which is not captured by slab ocean models (e.g., Boer and Yu, 2003).

Complementing the investigation by Rose and Ferreira (2013) and helping to understand the properties of warm equable climates, a subsequent study may focus on the role of latitudinal location of the peak OHT. In the present set of experiments, the peak oceanic transport was fixed at the latitude of $27^{\circ}$. Due to the local atmospheric compensation, this preferentially affects the atmospheric eddy transport of latent heat, which is dominant in this region. The overall response may be different if the OHT peak is located in regions where other components of the atmospheric heat transport are more important.
Another possible future line of investigation may deal with studying planets with different astrophysical parameters, such as rotation rate, eccentricity, and obliquity, with the goal of contributing to the rapidly growing field of the investigation of the atmospheres of exoplanets along the lines of some recent investigations (Pascale et al., 2013; Boschi et al., 2013; Lucarini et al., 2013). 


\section{Appendix A: The mean meridional circulation}

To analyze the mean meridional circulation we make use of the so-called Kuo-Eliassen equation (Kuo, 1956; Eliassen, 1951). This is a diagnostic equation which relates the mean meridional circulation (i.e., Hadley, Ferrel and Polar cell) to different sources.

Applying the quasi-geostrophic approximation and defining a stream function $\psi$ with

$$
\begin{aligned}
& {[v]=\frac{g}{2 \pi r \cos \phi} \frac{\partial \psi}{\partial p}} \\
& {[\omega]=-\frac{g}{2 \pi r \cos \phi} \frac{\partial \psi}{r \partial \phi},}
\end{aligned}
$$

the Kuo-Eliassen equation may be derived as (see Peixoto and Oort, 1992, Chap. 14.5.5)

$$
\begin{aligned}
\frac{f^{2} g}{2 \pi r \cos \phi} \frac{\partial^{2} \psi}{\partial p^{2}} & -\frac{g}{2 \pi r^{3} \rho[\theta]} \frac{\partial}{\partial \phi}\left(\frac{\partial[\theta]}{\partial p} \frac{1}{\cos \phi} \frac{\partial \psi}{\partial \phi}\right) \\
& =\frac{1}{r \rho[T]} \frac{\partial}{\partial \phi} \frac{[Q]}{c_{\mathrm{p}}}-f \frac{\partial[F]}{\partial p} \\
& -\frac{1}{r^{2} \rho[\theta]} \frac{\partial}{\partial \phi} \frac{\partial\left[v^{*} \theta^{*}\right] \cos \phi}{\cos \phi \partial \phi} \\
& +\frac{f}{r \cos ^{2} \phi} \frac{\partial^{2}\left[u^{*} v^{*}\right] \cos ^{2} \phi}{\partial p \partial \phi},
\end{aligned}
$$

where, in addition to the symbols defined above, $f$ is the Coriolis parameter, $\rho$ density, $\theta$ potential temperature, $Q$ diabatic heating and $F$ the tendency of the zonal wind due to friction.

We solve the Kuo-Eliassen equation for $\psi$ by applying an iterative method (Gauss-Seidel method) to its finite difference approximation. Thus, we are able to diagnose the contributions from the different sources to the mean meridional circulation, which are diabatic heating (1st term, righthand side), friction (2nd term, right-hand side), meridional eddy heat transport (3rd term, right-hand side) and eddy momentum transport (4th term, right-hand side). We note that, though the equation, in the present form, involves the quasigeostrophic approximation, it has been shown to be reasonably applicable even in the deep tropics (Kim and Lee, 2001a, b). In addition, as pointed out by Kim and Lee (2001a, b), it should be noted that this diagnostics will only yield the direct effect of the particular source. Since all processes are strongly interlinked, changes in one source will lead to changes in other terms. For example, according to the equations of motion changes in the meridional eddy momentum transport will affect the frictional dissipation of zonal mean momentum. These indirect effects cannot be identified with our (linear) methodology.

While the Kuo-Eliassen equation gives us the classical three-cell picture of the mean meridional circulation, the TEM formalism (Andrews and McIntyre, 1976) provides a closer link to the total atmospheric meridional heat transport.
Defining the residual stream function $\psi_{\text {res }}$ with

$$
\begin{aligned}
v_{\text {res }} & =\frac{g}{2 \pi r \cos \phi} \frac{\partial \psi_{\text {res }}}{\partial p} \\
\omega_{\text {res }} & =-\frac{g}{2 \pi r \cos \phi} \frac{\partial \psi_{\text {res }}}{r \partial \phi}
\end{aligned}
$$

and

$$
\begin{aligned}
& v_{\text {res }}=[v]-\frac{\partial}{\partial p}\left(\frac{\left[v^{*} \theta^{*}\right]}{\partial \theta_{\mathrm{s}} / \partial p}\right) \\
& \omega_{\text {res }}=[\omega]+\frac{1}{r \cos \phi} \frac{\partial}{\partial \phi}\left(\frac{\left[v^{*} \theta^{*}\right] \cos \phi}{\partial \theta_{\mathrm{s}} / \partial p}\right),
\end{aligned}
$$

an equation similar to the Kuo-Eliassen equation can be obtained for $\psi_{\text {res }}$.

$$
\begin{aligned}
\frac{f^{2} g}{2 \pi r \cos \phi} \frac{\partial^{2} \psi_{\text {res }}}{\partial p^{2}} & -\frac{g}{2 \pi r^{3} \rho[\theta]} \frac{\partial}{\partial \phi}\left(\frac{\partial \theta_{\mathrm{s}}}{\partial p} \frac{\partial \psi_{\text {res }}}{\cos \phi \partial \phi}\right) \\
& =\frac{1}{r \rho[T]} \frac{\partial}{\partial \phi} \frac{[Q]}{c_{\mathrm{p}}}-f \frac{\partial[F]}{\partial p} \\
& -\frac{f}{r \cos \phi} \frac{\partial(\operatorname{div} \boldsymbol{F})}{\partial p},
\end{aligned}
$$

with $\theta_{\mathrm{s}}$ denoting the constant, global mean potential temperature at a given pressure level according to quasi-geostrophic scaling.

Here, the total effect of the eddies on the meridional circulation (viewed from a Lagrangian perspective) is given by the divergence of the Eliassen-Palm flux $(\boldsymbol{F})$ with

$$
\begin{aligned}
& F_{\phi}=-r \cos \phi\left[u^{*} v^{*}\right] \\
& F_{\mathrm{p}}=f r \cos \phi \frac{\left[v^{*} \theta^{*}\right]}{\partial \theta_{\mathrm{s}} / \partial p} .
\end{aligned}
$$

The TEM residual mean circulation represents the part of the mean meridional circulation which is not balanced by the convergence of the eddy heat transport. It is qualitatively similar to the dry isentropic circulation with the important difference that the TEM circulation does not close at the surface (Held and Schneider, 1999).

Though representing a different view of the circulation, and, in particular of the role of the eddies, the Kuo-Eliassen equation and the TEM equation represent the same physics. This can be seen by rearranging the terms of the KuoEliassen or the TEM equation (and neglecting differences between globally and zonally averaged stability) to give 


$$
\begin{aligned}
\frac{f^{2} g}{2 \pi r \cos \phi} \frac{\partial^{2} \psi}{\partial p^{2}} & -\frac{g}{2 \pi r^{3} \rho[\theta]} \frac{\partial}{\partial \phi}\left(\frac{\partial[\theta]}{\partial p} \frac{1}{\cos \phi} \frac{\partial \psi}{\partial \phi}\right) \\
& +\frac{f^{2} g}{2 \pi r \cos \phi} \frac{\partial^{2} \psi_{\mathrm{E}}}{\partial p^{2}}-\frac{g}{2 \pi r^{3} \rho[\theta]} \frac{\partial}{\partial \phi} \\
& \left(\frac{\partial[\theta]}{\partial p} \frac{1}{\cos \phi} \frac{\partial \psi_{\mathrm{E}}}{\partial \phi}\right)=\frac{1}{r \rho[T]} \frac{\partial}{\partial \phi} \frac{[Q]}{c_{\mathrm{p}}} \\
& -f \frac{\partial[F]}{\partial p}-\frac{f}{r \cos \phi} \frac{\partial(\operatorname{div} \boldsymbol{F})}{\partial p} .
\end{aligned}
$$

We see that considering the combined effect of eddy heat and momentum transport leads to a second circulation defined by the eddy heat transport:

$\psi_{\mathrm{E}}=-\frac{2 \pi r \cos \phi}{g} \frac{\left[v^{*} \theta^{*}\right]}{\partial \theta_{\mathrm{S}} / \partial p}$,

where $\psi_{\mathrm{E}}$ is sometimes referred to as the Stokes stream function.

Czaja and Marshall (2006) showed that the atmospheric heat transport can be represented by the product of a moist TEM residual circulation and the vertical contrast in moist static energy (or equivalent potential temperature $\theta_{\mathrm{e}}$ ) if both the eddy transport and the vertical gradient of $\theta$ are replaced by the respective values utilizing $\theta_{\mathrm{e}}$. Unfortunately, as pointed out by Pauluis et al. (2011) and Laliberté and Pauluis (2010), there is no simple way to represent a welldefined moist isentropic circulation in the latitude-pressure plane. Therefore, we additionally investigate the mean circulation on dry and moist isentropes by interpolating the mass transport to levels of constant $\theta$ and $\theta_{\mathrm{e}}$, respectively.

\section{Appendix B: Nonequilibrium thermodynamics}

Let $\Omega$ be the volume domain of the climate system and $\dot{Q}$ be the local heating rate due to frictional dissipation and convergence of heat fluxes including radiative, sensible and latent heat components. At each instant $t$ we divide $\Omega$ into two subsections, so that $Q(x, t)>0, x \in \Omega^{+}$ defining $Q^{+}$, and $Q(x, t)<0, x \in \Omega^{-}$for $Q^{-}$. We wish to remark that the domains $\Omega^{+}$and $\Omega^{-}$are time dependent. Integrating the two heating components results in $\int_{\Omega^{+}} \rho \dot{Q}^{+} \mathrm{d} V+\int_{\Omega^{-}} \rho \dot{Q}^{-} \mathrm{d} V=\dot{\Phi}^{+}+\dot{\Phi}^{-}$. Johnson (2000) and Lucarini (2009) show that the time average $\overline{\dot{\Phi}^{+}}+\overline{\dot{\Phi}^{-}}$ gives the rate of generation of available potential energy, so that

$\overline{\dot{W}}=\overline{\dot{\Phi}^{+}}+\overline{\dot{\Phi}^{-}}=\eta \overline{\dot{\Phi}^{+}}$,

where the efficiency of the climate machine can be expressed as

$\eta=\frac{\overline{\dot{\Phi}^{+}}+\overline{\dot{\Phi}^{-}}}{\overline{\dot{\Phi}^{+}}}$.
This expression represents the ratio for the work output $\overline{\dot{\Phi}^{+}}+\overline{\dot{\Phi}^{-}}$to the heat input $\overline{\dot{\Phi}^{+}}$. At each instant one defines the quantities $\Sigma^{+(-)}=\frac{\int_{\Omega^{+(-)}} \rho \dot{Q}^{+(-)}}{\int_{\Omega^{+(-)}} T}$, which are the instantaneous entropy sources and sinks in the system. As explained in Johnson (2000) and Lucarini (2009), we have $\overline{\dot{\Sigma}^{+}}+\overline{\dot{\Sigma}^{-}}=0$. We can then introduce the scale temperatures $\Theta^{+}=\frac{\overline{\Phi^{+}}}{\dot{\Sigma}^{+}}$and $\Theta^{-}=\frac{\overline{\Phi^{-}}}{\dot{\Sigma}^{-}}$, so that Eq. (B2) can be rewritten as $\eta=\frac{\Theta^{+}-\Theta^{-}}{\Theta^{+}}$,

where $\Theta^{+}>\Theta^{-}$.

Hence, the motion of the general circulation of the system can be sustained against friction because zones being already relatively warm absorb heat, whereas the relatively low-temperature zones are cooled.

The Lorenz energy cycle can thus be seen as resulting from the work of an equivalent Carnot engine operating between the two (dynamically determined) reservoirs at temperatures $\Theta^{+}$and $\Theta^{-}$. However, the climate is far from being a perfect engine, as many irreversible processes take place; nonetheless, a Carnot-equivalent picture can be drawn as described.

Let us now delve into such irreversible processes. In the climate system two rather different sets of processes contribute to the total entropy production (Peixoto and Oort, 1992; Goody, 2000; Ambaum, 2010). The first set of processes is responsible for the irreversible thermalization of photons emitted near the Sun's corona at roughly $5800 \mathrm{~K}$, absorbed and then re-emitted at much lower temperatures, typical of the Earth's climate $(\sim 255 \mathrm{~K})$. This gives the largest contribution to the total average rate of entropy production for the Earth system of about $900 \mathrm{~mW} \mathrm{~m}^{-2} \mathrm{~K}^{-1}$ (Peixoto and Oort, 1992; Ambaum, 2010). The remaining contribution is due to the processes responsible for mixing and diffusion inside the fluid component of the Earth system and for the dissipation of kinetic energy due to viscous processes. This constitutes the so-called material entropy production and is considered to be the entropy-related quantity of main interest as far as the properties of the climate system are concerned. Further relevant research on entropy production in the climate system treating also the geochemical and radiative contribution to entropy production can be found in Kleidon (2009) and Wu and Liu (2010), respectively.

The entropy budget of geophysical fluids at steady state, following Goody (2000) and Lucarini et al. (2011), is given by

$\overline{\dot{S}(\Omega)}=\int_{\Omega} \rho\left(\overline{\frac{\dot{q}_{\text {rad }}}{T}}+\overline{\dot{s}_{\text {mat }}}\right) \mathrm{d} V=0$,

where $\dot{q}_{\text {rad }}$ indicates the heating rate by the convergence of radiative fluxes, $T$ is the local temperature at which the energy is gained or lost, while $\dot{s}_{\text {mat }}$ represents the density of 
entropy production associated with the irreversibility of processes involving the fluid medium. Equation (B4) represents the entropy budget and states that in a steady state the radiative entropy source must be balanced by the rate of material entropy production $\dot{S}_{\text {mat }}$ due to material irreversible processes. A detailed discussion of this aspect is found in Lucarini and Pascale (2014), where the contributions to the material entropy production on various spatial and temporal scales are discussed.

In a steady-state climate the material entropy production $\dot{S}_{\text {mat }}(\Omega)$ can be expressed in general terms as

$$
\begin{aligned}
\overline{\dot{S}_{\text {mat }}}(\Omega) & =\int_{\Omega} \rho \overline{\dot{s}_{\text {mat }}} \mathrm{d} V=\int_{\Omega} \overline{\frac{\varepsilon^{2}}{T}} \mathrm{~d} V \\
& +\int_{\Omega} \overline{\left(\boldsymbol{F}_{\text {sens }}+\boldsymbol{F}_{\text {lat }}\right) \cdot \nabla \frac{1}{T}} \mathrm{~d} V=-\int_{\Omega} \rho \frac{\overline{\dot{q}_{\mathrm{rad}}}}{T} \mathrm{~d} V,
\end{aligned}
$$

where $\overline{\dot{s}_{\text {mat }}}$ is the time-averaged density of entropy production due to the following irreversible processes inside the medium: dissipation of kinetic energy $\left(\varepsilon^{2}\right.$ is the specific dissipation rate) and turbulent transport of heat down the temperature gradient ( $\boldsymbol{F}_{\text {sens }}$ and $\boldsymbol{F}_{\text {lat }}$ being the sensible and latent turbulent heat fluxes, respectively).

One needs to underline that a more refined treatment of the entropy production related to the hydrological cycle has been proposed by, e.g., Pauluis and Held (2002a, b) and Romps (2008). Nonetheless, as discussed in detail in Lucarini et al. (2014), the overall contribution of the entropy production due to the hydrological cycle can be reconstructed in a fundamentally correct way also in the simplified method proposed here, where one needs not to follow all the complicated irreversible processes related to the hydrological cycle evaporation of liquid water in unsaturated air, condensation of water vapor in supersaturated air, and molecular diffusion of water vapor.

Note that one can compute the entropy production as

$$
\begin{aligned}
\overline{\dot{S}_{\text {mat }}(\Omega)} & =\int_{\Omega} \overline{\frac{\varepsilon^{2}}{T}} \mathrm{~d} V+\int_{\Omega} \frac{\overline{-\boldsymbol{\nabla} \cdot\left(\boldsymbol{F}_{\text {sens }}+\boldsymbol{F}_{\text {lat }}\right)}}{T} \mathrm{~d} V \\
& +\int_{\partial \Omega} \frac{\overline{\boldsymbol{F}_{\text {sens }}+\boldsymbol{F}_{\text {lat }}}}{T} \cdot \hat{n} \mathrm{~d} S,
\end{aligned}
$$

where the first term is unchanged, the second terms describes the entropy gain and loss due to heating and cooling by convergence of sensible and latent turbulent heat fluxes, and the last term is the net entropy flux across the boundaries of $\Omega$. If one considers the atmospheric domain as $\Omega$, such a term becomes equal to the integral at the surface of the ratio between the sum of the sensible and of the latent heat flux divided by the surface temperature. Equation (B6) represents the way entropy production is typically computed in numerical models. If one considers the whole climate system as $\Omega$, the boundary terms disappear. Nonetheless, another term proportional to a Dirac's delta at $z=z_{\text {surf }}=0$ appears, resulting from the divergence of the turbulent flux due to the net evaporation at the surface. If we integrate over $\Omega$, corresponding to the whole climate system, the contribution of this term is exactly the same as in the case where $\Omega$ corresponds to the atmosphere only. In other terms, our simplified, non-dynamical representation of the ocean is such that all the entropy is produced in the atmosphere.

We can now separate in Eq. (B5) - or, equivalently, in Eq. (B6) the first term from the rest, so that, following Lucarini (2009), the material entropy production can be expressed as

$\overline{\dot{S}_{\text {mat }}(\Omega)}=\overline{\dot{S}_{\min }(\Omega)}+\overline{\dot{S}_{\text {exc }}(\Omega)}$,

where $\overline{\dot{S}}_{\min }(\Omega)$ is the minimum value of entropy production compatible with the presence of the average dissipation rate $\int \epsilon^{2} \mathrm{~d} V$, while $\overline{\dot{S}_{\text {exc }}(\Omega)}$ is the excess of entropy production $\Omega$ with respect to such a minimum. One can associate $\overline{\dot{S}_{\text {min }}}$ exactly with the term in Eq. (B5) related to the dissipation of kinetic energy, while $\overline{\dot{S}_{\text {exc }}}$ can be identified with the sum of the other two terms.

If we take the ratio of the two terms on the right-hand side in Eq. (B7), we have

$$
\alpha=\frac{\overline{\dot{S}_{\text {exc }}(\Omega)}}{\dot{S}_{\text {min }}(\Omega)} \approx \frac{\int_{\Omega} \overline{\left(\boldsymbol{F}_{\text {sens }}+\boldsymbol{F}_{\text {lat }}\right) \cdot \nabla \frac{\mathbf{1}}{\boldsymbol{T}} \mathrm{d} V}}{\int_{\Omega} \frac{\varepsilon^{2}}{T} \mathrm{~d} V},
$$

where $\alpha$ is the degree of irreversibility (Lucarini, 2009) and determines the ratio between the contributions to entropy production by down-gradient turbulent transport and by viscous dissipation of mechanical energy. If this ratio is close to $0(\alpha \rightarrow 0)$, all the production of entropy is exclusively caused by unavoidable viscous dissipation. If the turbulent heat transport in the system from high- to low-temperature regions is enhanced, then entropy production also increases. However, if the turbulent heat transport down the temperature gradient is maximized, the efficiency declines, since the temperature difference between the warm and cold reservoirs tends to become 0 . The characterization of the maximum entropy production principle (MEPP) suggests that the climate system adjusts in such a way as to maximize the entropy production (Paltridge, 1975; Grassl, 1981; Kleidon and Lorenz, 2005).

\section{Appendix C: The Lorenz energy cycle}

The atmospheric energy cycle proposed by Lorenz (1955) is one of the most important concepts for understanding the global atmospheric circulation by means of energy conservation and by considering the integrated effects of physical 
mechanisms involved, e.g., the generation of available potential energy by external forcing, the dissipation of kinetic energy and the energy conversions by baroclinic and barotropic processes. At the same time the Lorenz energy cycle gives information about the relative importance of the zonal mean circulation, the eddies and the interaction between both.

Referring to the reservoirs of zonal available potential energy, eddy available potential energy, zonal kinetic energy and eddy kinetic energy as $P_{\mathrm{M}}, P_{\mathrm{E}}, K_{\mathrm{M}}$ and $K_{\mathrm{E}}$, respectively, the Lorenz energy cycle (i.e., the budget equations) may be written as

$$
\begin{aligned}
\frac{\mathrm{d} P_{\mathrm{M}}}{\mathrm{d} t} & =\left[S_{\mathrm{P}}\right]-C\left(P_{\mathrm{M}}, P_{\mathrm{E}}\right)-C\left(P_{\mathrm{M}}, K_{\mathrm{M}}\right) \\
\frac{\mathrm{d} P_{\mathrm{E}}}{\mathrm{d} t} & =S_{\mathrm{P}}^{*}+C\left(P_{\mathrm{M}}, P_{\mathrm{E}}\right)-C\left(P_{\mathrm{E}}, K_{\mathrm{E}}\right) \\
\frac{\mathrm{d} K_{\mathrm{E}}}{\mathrm{d} t} & =S_{\mathrm{K}}^{*}+C\left(P_{\mathrm{E}}, K_{\mathrm{E}}\right)-C\left(K_{\mathrm{E}}, K_{\mathrm{M}}\right) \\
\frac{\mathrm{d} K_{\mathrm{M}}}{\mathrm{d} t} & =\left[S_{\mathrm{K}}\right]+C\left(K_{\mathrm{E}}, K_{\mathrm{M}}\right)+C\left(P_{\mathrm{M}}, K_{\mathrm{M}}\right),
\end{aligned}
$$

where $\left[S_{\mathrm{P}}\right], S_{\mathrm{P}}^{*},\left[S_{\mathrm{K}}\right]$ and $S_{\mathrm{K}}^{*}$ are external sources and/or sinks of the respective quantities and $C(A, B)$ denotes the conversion from $A$ to $B$.

To compute the individual contributions, we follow the work of Ulbrich and Speth (1991). In pressure coordinates, the reservoirs are given by

$$
\begin{aligned}
P_{\mathrm{M}} & =\left\langle\frac{\gamma}{2}([T]-\{T\})^{2}\right\rangle \\
P_{\mathrm{E}} & =\left\langle\frac{\gamma}{2}\left[T^{* 2}\right]\right\rangle \\
K_{\mathrm{M}} & =\left\langle\frac{1}{2}\left([u]^{2}+[v]^{2}\right)\right\rangle \\
K_{\mathrm{E}} & =\left\langle\frac{1}{2}\left(\left[u^{* t 2}\right]+\left[v^{* 2}\right]\right)\right\rangle,
\end{aligned}
$$

and the conversion terms are

$$
\begin{gathered}
C\left(P_{\mathrm{M}}, P_{\mathrm{E}}\right)=-\langle\gamma \\
\ldots-\frac{R}{p \cdot c_{\mathrm{p}}}([T]-\{T\}) \\
C\left(P_{\mathrm{M}}, K_{\mathrm{M}}\right)=-\left\langle\frac{R}{p}([\omega]-\{\omega\})\left(\left[T_{\mathrm{v}}\right]-\left\{T_{\mathrm{v}}\right\}\right)\right\rangle \\
C\left(P_{\mathrm{E}}, K_{\mathrm{E}}\right)=-\left\langle\frac{R}{p}\left[\omega^{*} T_{\mathrm{v}}^{*}\right]\right\rangle \\
C\left(K_{\mathrm{M}}, K_{\mathrm{E}}\right)=\left\langle\left(\left[u^{*} v^{*}\right] \frac{\partial[u]}{r \partial \phi}+\left[u^{*} v^{*}\right][u] \frac{\tan \phi}{r}+\left[v^{*} v^{*}\right]\right.\right. \\
\left.\left.\frac{\partial[v]}{r \partial \phi}-\left[u^{*} u^{*}\right][v] \frac{\tan \phi}{r}+\left[\omega^{*} u^{*}\right] \frac{\partial[u]}{\partial p}+\left[\omega^{*} v^{*}\right] \frac{\partial[v]}{\partial p}\right)\right\rangle,
\end{gathered}
$$

where $[x]$ is the zonal mean; $x^{*}$ is the deviation from zonal mean; $\{x\}$ is the global horizontal mean; $\langle x\rangle=\frac{1}{g \cdot A} \int_{A} \int_{p} x \mathrm{~d} p \mathrm{~d} A ; A$ is the horizontal area; $c_{\mathrm{p}}$ is the specific heat at constant pressure; $g$ is gravity; $p$ is pressure; $r$ is the radius of the Earth; $R$ is a gas constant; $T$ is temperature; $T_{\mathrm{v}}$ is virtual temperature; $u$ is zonal wind; $v$ is meridional wind; $\omega$ is vertical ( $p$ ) velocity; $\phi$ is latitude; and $\gamma$ is the stability parameter $=-\frac{R}{p}\left(\frac{\partial[\bar{T}]}{\partial p}-\frac{R}{c_{\mathrm{p}}} \frac{[\bar{T}]}{p}\right)^{-1}$.

The external sources and/or sinks are diagnosed from the respective residuals. We note that in Ulbrich and Speth (1991) these energetics were formulated for a mixed spacetime domain. In our case, however, the contributions by stationary eddies are 0 because of the zonally symmetric forcing.

We also note that by using above equations the computed annual averaged values include contributions from the annual cycle. It turns out, however, that only the reservoirs $P_{\mathrm{M}}$ and $K_{\mathrm{M}}$ and the conversion $C\left(P_{\mathrm{M}}, K_{\mathrm{M}}\right)$ are affected. 
Acknowledgements. The authors wish to acknowledge support by the Cluster of Excellence CliSAP. V. Lucarini wishes to acknowledge the financial support provided by the FP7 ERC-Starting Investigator Grant NAMASTE - Thermodynamics of the Climate System (Grant no. 257106). We thank the anonymous reviewers for constructive criticism and, in particular, F. Laliberté for his thorough evaluation and his valuable suggestions. The preparation of this work greatly benefitted from interactions with D. Battisti, J. Marshall, O. Pauluis, T. Schneider, and P. Stone.

Edited by: D. Kirk-Davidoff

\section{References}

Ambaum, M. H. P. M.: Thermal physics of the atmosphere, vol. 1, J. Wiley \& Sons, Chichester, 2010.

Andrews, D. G. and McIntyre, M. E.: Planetary waves in horizontal and vertical shear: The generalized Eliassen-Palm relation and the mean zonal acceleration, J. Atmos. Sci., 33, 2031-2048, 1976.

Barreiro, M., Cherchi, A., and Masina, S.: Climate sensitivity to changes in ocean heat transport, J. Climate, 24, 5015-5030, 2011.

Boer, G. J. and Yu, B.: Dynamical aspects of climate sensitivity, Geophys. Res. Lett., 30, 1135-1139, 2003.

Boschi, R., Lucarini, V., and Pascale, S.: Bistability of the climate around the habitable zone: a thermodynamic investigation, Icarus, 226, 1724-1742, 2013.

Collins, M., An, S.-I., Cai, W., Ganachaud, A., Guilyardi, E., Jin, F.F., Jochum, M., Lengaigne, M., Power, S., Timmermann, A., Vecchi, G., and Wittenberg, A.: The impact of global warming on the tropical Pacific Ocean and El Niño, Nat. Geosci., 3, 391397, 2010.

Czaja, A. and Marshall, J.: The partitioning of poleward heat transport between the atmosphere and ocean, J. Atmos. Sci., 63, 1498-1511, 2006

Eliassen, A.: Slow frictionally controlled meridional circulation in a circular vortex, Astro. Norv., 5, 19-60, 1951.

Enderton, D. and Marshall, J.: Explorations of atmosphere-oceanice climates on an aquaplanet and their meridional energy transports, J. Atmos. Sci., 66, 1593-1610, 2008.

Fedorov, A. V., Dekens, P. S., McCarthy, M., Ravelo, A. C., deMenocal, P. B., Barreiro, M., Pacanowski, R. C., and Philander, S. G.: The Pliocene Paradox (Mechanisms for a permanent El Niño), Science, 312, 1485-1489, 2006.

Fraedrich, K., Jansen, H., Kirk, E., Luksch, U., and Lunkeit, F.: The Planet Simulator: Towards a user friendly model, Meteorol. Z., 14, 299-304, 2005.

Goody, R.: Sources and sinks of climate entropy, Q. J. Roy. Meteorol. Soc., 126, 1953-1970, 2000.

Grassl, H.: The climate at maximum entropy production by meridional atmospheric and oceanic heat fluxes, Q. J. Roy. Meteorol. Soc., 107, 153-166, 1981.

Held, I. M.: The partitioning of the poleward energy transport between the tropical ocean and atmosphere, J. Atmos. Sci., 58, 943-948, 2001.

Held, I. M. and Schneider, T.: The surface branch of the zonally averaged mass transport circulation in the troposphere, J. Atmos. Sci., 56, 1688-1697, 1999.
Herweijer, C., Seager, R., Winton, M., and Clement, A.: Why ocean heat transport warms the global mean climate, Tellus A, 57, 662$675,2005$.

Hoskins, B. J. and Simmons, A. J.: A multi-layer spectral method and the semi-implicit method, Q. J. Roy. Meteorol. Soc., 101, 637-655, 1975.

IPCC: Climate Change 2013: The Physical Science Basis, in: Contribution of Working Group I to the Fifth Assessment Report of the Intergovernmental Panel on Climate Change, edited by: Stocker, T. F., Qin, D., Plattner, G.-K., Tignor, M., Allen, S. K., Boschung, J., Nauels, A., Xia, Y., Bex, V., and Midgley, P. M., Cambridge University Press, Cambridge, UK and New York, NY, USA, 1535 pp., 2013.

James, I. N. and Gray, L. J.: Concerning the effect of surface drag on the circulation of a planetary atmosphere, Q. J. Roy. Meteorol. Soc., 112, 1231-1250, 1986.

Johnson, D. R.: Entropy, the Lorenz energy cycle and climate, in: General Circulation Model Development: Past, Present and Future, chapter 22, Academic Press, San Diego, London, 659-720, 2000.

Kim, H.-K. and Lee, S.: Hadley cell dynamics in a primitive equation model, Part I: Axisymmetric flow, J. Atmos. Sci., 58, 28452858, 2001a.

Kim, H.-K. and Lee, S.: Hadley cell dynamics in a primitive equation model, Part II: Nonaxisymmetric flow, J. Atmos. Sci., 58, 2859-2871, 2001b.

Kleidon, A.: Nonequilibrium thermodynamics and maximum entropy production in the Earth system, Naturwissenschaften, 96, 653-677, 2009.

Kleidon, A. and Lorenz, R.: Entropy production by Earth system processes, in: thermodynamics and the production of entropy, Springer, Heidelberg, 2005.

Koll, D. D. B. and Abbot, D. S.: Why tropical sea surface temperature is insensitive to ocean heat transport changes, J. Climate, 26, 6742-6749, 2013.

Kuo, H.-L.: Forced and free meridional circulations in the atmosphere, J. Meteorol., 13, 561-568, 1956.

Kuo, H.-L.: On formation and intensification of tropical cyclones through latent heat release by cumulus convection, J. Atmos. Sci., 22, 40-63, 1965.

Kuo, H.-L.: Further studies of the parameterization of the influence of cumulus convection on large-scale flow, J. Atmos. Sci., 31, 1232-1240, 1974.

Lacis, A. A. and Hansen, J. E.: A parameterization for the absorption of solar radiation on the Earth's atmosphere, J. Atmos. Sci., 31, 118-133, 1974.

Laliberté, F. and Pauluis, O.: Winter intensivication of the moist branch of the circulation of the 21 st century climate, Geophys. Res. Lett., 37, L20707, doi:10.1029/2010GL045007, 2010.

Laliberté, F., Shaw, T. A., and Pauluis, O.: Moist recirculation and water vapor transport on dry isentropes, J. Atmos. Sci., 69, 875890, 2012.

Laliberté, F., Zika, J., Mudryk, L., Kushner, P. J., Kjellsson, J., and Döös, K.: Constrained work output of the moist atmospheric heat engine in a warming climate, Science, 347, 540-543, 2015.

Laursen, L. and Eliasen, E.: On the effects of the damping mechanisms in an atmospheric general circulation model, Tellus A, 41, 385-400, 1989. 
Lorenz, E. N.: Available potential energy and the maintenance of the general circulation, Tellus, 7, 157-167, 1955.

Lorenz, E. N.: The nature and theory of the general circulation of the atmosphere, World Meteorological Organization, Geneva, 1967.

Louis, J. F.: A parametric model of vertical eddy fluxes in the atmosphere, Bound.-Lay. Meteorol., 17, 187-202, 1979.

Louis, J. F., Tiedtke, M., and Geleyn, M.: A short history of the PBL parameterisation at ECMWF, in: Proceedings, ECMWF Workshop on Planetary Boundary Layer Parameterization, 2527 November 1981, Reading, 59-80, 1982.

Lu, J., Vecchi, G. A., and Reichler, T.: Expansion of the Hadley cell under global warming, Geophys. Res. Lett., 34, L06805, doi:10.1029/2006GL028443, 2007.

Lucarini, V.: Thermodynamic efficiency and entropy production in the climate system, Phys. Rev. E, 80, 021118, doi:10.1103/PhysRevE.80.021118, 2009.

Lucarini, V. and Pascale, S.: Entropy production and coarse graining of the climate fields in a general circulation model, Clim. Dynam., 43, 981-1000, 2014.

Lucarini, V. and Ragone, F.: Energetics of climate models: net energy balance and meridional enthalpy transport, Rev. Geophys., 49, RG1001, doi:10.1029/2009RG000323, 2011.

Lucarini, V., Fraedrich, K., and Lunkeit, F.: Thermodynamics of climate change: generalized sensitivities, Atmos. Chem. Phys., 10, 9729-9737, doi:10.5194/acp-10-9729-2010, 2010a.

Lucarini, V., Fraedrich, K., and Lunkeit, F.: Thermodynamic analysis of snowball earth hysteresis experiment: efficiency, entropy production, and irreversibility, Q. J. Roy. Meterol. Soc., 136, 2$11,2010 \mathrm{~b}$.

Lucarini, V., Fraedrich, K., and Ragone, F.: New results on the thermodynamical properties of the climate system, J. Atmos. Sci., 68, 2438-2458, 2011.

Lucarini, V., Pascale, S., Boschi, R., Kirk, E., and Iro, N.: Habitability and multistablility in earth-like planets, Astron. Nachr., 334, 576-588, 2013.

Lucarini, V., Blender, R., Pascale, S., Ragone, F., Wouters, J., and Herbert, C.: Mathematical and Physical Ideas for Climate Science, Rev. Geophys., 52, 809-859, 2014.

Paltridge, G.: Global dynamics and climate - a system of minimum entropy exchange, Q. J. Roy. Meteorol. Soc., 101, 475-484, 1975.

Pascale, S., Ragone, F., Lucarini, V., Wang, Y., and Boschi, R.: Nonequilibrium thermodynamics of circulation regimes in optically-thin, dry atmospheres, Planet. Space Sci., 84, 48-65, 2013.

Pauluis, O. and Held, I.: Entropy budget of an atmosphere in radiative convective equilibrium, Part I: Maximum work and frictional dissipation, J. Atmos. Sci., 59, 125-139, 2002a.

Pauluis, O. and Held, I.: Entropy budget of an atmosphere in radiative convective equilibrium, Part II: Latent heat transport and moist processes, J. Atmos. Sci., 59, 140-149, 2002 b.
Pauluis, O., Shaw, T., and Laliberté, F.: A statistical generalization of the transformed Eulerian-mean circulation far an arbitrary vertical coordinate system, J. Atmos. Sci., 69, 1766-1783, 2011.

Peixoto, J. P. and Oort, A. H.: Physics of Climate, American Institute of Physics, New York, 1992.

Rind, D. and Chandler, M.: Increased ocean heat transports and warmer climate, J. Geophys. Res., 96, 7437-7461, 1991.

Roeckner, E., Arpe, K., Bengtsson, L., Brinkop, S., Dümenil, L., Esch, M., Kirk, E., Lunkeit, F., Ponater, M., Rockel, B., Sausen, R., Schlese, U., Schubert, S., and Windelband, M.: Simulation of the present-day climate with the ECHAM-3 model: impact of model physics and resolution, Meteorologie, Report No. 93, Max-Planck Institut für Meteorologie, Hamburg, 171 pp., 1992

Romanova, V., Lohmann, G., Grosfeld, K., and Butzin, M.: The relative roles of oceanic heat transport and orography on glacial climate, Quaternary Sci. Rev., 25, 832-845, 2006.

Romps, D. M.: The dry-entropy budget of a moist atmosphere, J. Atmos. Sci., 65, 3779-3799, 2008.

Rose, B. and Ferreira, D.: Ocean heat transport and water vapor greenhouse in a warm equable climate: a new look at the low gradient paradox, J. Climate, 26, 2117-2136, doi:10.1175/JCLID-11-00547.1, 2013.

Sasamori, T.: The radiative cooling calculation for application to general circulation experiments, J. Appl. Meteorol., 7, 721-729, 1968.

Slingo, A. and Slingo, J. M.: Response of the National Center for Atmospheric Research community climate model to improvements in the representation of clouds, J. Geophys. Res., 96, 341357, 1991.

Sloan, L. C., Walker, J. C. G., and Moore Jr., T. C.: Possible role of oceanic heat transport in early Eocene climate, Paleoceanography, 10, 347-356, 1995.

Stephens, G. L.: Radiation profiles in extended water clouds, II: Parameterization schemes, J. Atmos. Sci., 34, 2123-2132, 1978.

Stephens, G. L., Ackermann, S., and Smith, E. A.: A shortwave parameterization revised to improve cloud absorption, J. Atmos. Sci., 41, 687-690, 1984.

Stone, P. H.: Constraints on dynamical transports of energy on a spherical planet, Dynam. Atmos. Oceans, 2, 123-139, 1978.

Ulbrich, U. and Speth, P.: The global energy cycle of stationary and transient atmospheric waves: results from ECMWF analyses, Meteorol. Atmos. Phys., 45, 125-138, 1991.

Winton, M.: On the climate impact on ocean circulation, J. Climate, 16, 2875-2889, 2003.

$\mathrm{Wu}, \mathrm{W}$. and Liu, Y.: Radiation entropy flux and entropy production of the Earth system, Rev. Geophys., 48, 1-27, 2010. 